\title{
$R$-LRFD: Load and Resistance Factor Design Considering Robustness
}

\author{
Wenping Gong ${ }^{1}$, C. Hsein Juang ${ }^{2}$, Sara Khoshnevisan ${ }^{3}$, and Kok-Kwang Phoon ${ }^{4}$
}

\section{Abstract}

LRFD (Load and Resistance Factor Design) is becoming a design method of choice in geotechnical practice, in lieu of the factor of safety (FS)-based design approach. However, even with LRFD, the need to reduce the variation in the predicted performance of a geotechnical system (or a geotechnical structure), referred to herein as the system response under applied loads, is still apparent. This paper presents a novel approach for applying existing LRFD codes, with explicit consideration of design robustness, safety, and cost efficiency. The recently developed reliability-based robust geotechnical design (RGD) approach is modified such that it can be integrated with the standard LRFD approach. This modified RGD approach is termed $R$ LRFD, which stands for Robust Load and Resistance Factor Design. In $R$-LRFD, the robustness of the system response against the variation in uncertain parameters is explicitly considered. Unlike the reliability-based design (RBD), the user is not required to conduct a full statistical characterization of uncertain parameters. With $R$-LRFD, the gain in the robustness, as reflected by the reduction in the sensitivity of the system response to the recognized but unquantified uncertainty of input parameters, is accompanied by an increase in cost. Thus, the concepts of Pareto front and knee point are introduced to aid in making an informed design decision. Further, a simplified procedure is developed to identify the most preferred design (knee point) in the design space, which is generally solved with multi-objective optimization algorithms. The effectiveness and significance of the proposed $R$-LRFD approach are demonstrated with 
24 two examples: one is the design of drilled shaft in sand (illustrated with a discrete design space)

25 and the other is the design of drilled shaft in clay (illustrated with a continuous design space).

27 Keywords: LRFD; Robust design; Sensitivity; Pareto front; Knee point; Drilled shaft.

${ }^{1}$ Research Assistant Professor, Glenn Department of Civil Engineering, Clemson University, Clemson, SC 29634. (Email: wenping@ clemson.edu)

${ }^{2}$ Professor, Glenn Department of Civil Engineering, Clemson University, Clemson, SC 29634; Department of Civil Engineering, National Central University, Taoyuan 320, Taiwan. (Corresponding author; email: hsein@clemson.edu)

${ }^{3}$ Research Assistant, Glenn Department of Civil Engineering, Clemson University, Clemson, SC 29634. (Email: skhoshn@g.clemson.edu)

${ }^{4}$ Professor, Department of Civil and Environmental Engineering, National University of 


\section{Introduction}

The uncertainties in soil parameters, solution model, applied loads, and those caused by the construction, often make it difficult to ascertain the performance of a geotechnical design. In traditional deterministic approaches, these uncertainties are not explicitly characterized and included in the design analysis; rather, a conservative factor of safety (FS) is adopted based on the concept of "calculated risk" (Casagrande 1965). This FS-based design approach often leads to an inefficient over-design with an unknown and/or inconsistent safety level, although underdesign is also a possibility. To achieve a more rational and consistent assessment of the safety, the reliability-based design (RBD) approach has long been suggested as an alternative (e.g., Honjo et al. 2000; Baecher and Christian 2003; Christian 2004; Phoon et al. 2003a\&b; Ang and Tang 2007; Fenton and Griffiths 2008; Najjar and Gilbert 2009; Park et al. 2012; Low and Phoon 2015). The RBD approach for the design of a geotechnical structure is often implemented with a target reliability index or failure probability, which is derived from a costbenefit analysis that balances investment and risk considering the failure probability and consequence.

In the context of RBD approach, the performance of a geotechnical structure is analyzed using probabilistic methods that consider explicitly uncertainties in input parameters and/or solution models. It is noted that although various methods have been investigated to estimate the statistics of soil parameters (e.g., Wang and Cao 2013; Cao and Wang 2014) and model errors (e.g., Zhang et al. 2009\&2010), the statistics of soil parameters and those of model errors could not be characterized with certainty due to limited availability of site-specific data. Because of the difficulty in obtaining the accurate statistical characterization of soil parameters and model errors in practice, the RBD approach is not widely applied in geotechnical practice; 
67 rather, the load and resistance factor design (LRFD) approach, which is a simpler variant of the

68 RBD approach by design, is more commonly used. The LRFD code employs partial factors

69 (e.g., resistance factors and load factors), which have been calibrated to achieve a target

70 reliability index approximately over a range of design scenarios covered by the code. From a 71 user's perspective, the partial factors for a given geotechnical design problem using a given 72 solution model are specified in the code (e.g., NSAI 2005; BSI 2007; NEN 2007; Standards 73 Australia 2009; AASHTO 2014); then, the main task of the design involves a proper selection 74 of the nominal values of uncertain parameters (e.g., resistance term and load term). The 75 resulting design is a function of the specified partial factors and selected nominal values, with 76 due consideration of cost (Honjo and Amatya 2005). The application of LRFD is identical to 77 that of the FS-based design approach, involving iterative adjustment of the geometries and 78 other design parameters until an economic design that satisfies safety requirements is achieved.

As is well recognized, LRFD is meant to be a simpler variant of the more demanding RBD; the ideal outcome is that the design obtained by LRFD could achieve the same target 81 reliability index as that obtained by RBD. However, the standard LRFD approach that involves 82 fixed partial factors cannot cover all design scenarios involving different levels of variation of 83 soil parameters and model errors. For a given design scenario, the use of the standard LRFD 84 code may lead to a design that deviates from the target reliability index by an unknown amount, 85 more likely on the conservative side but under-design is also a possibility. This drawback was 86 well recognized (e.g., Honjo et al. 2002; Phoon et al. 2003a; Paikowsky et al. 2004; Honjo and 87 Amatya 2005; Low 2005; Najjar and Gilbert 2009; Stuedlein et al. 2012; Park et al. 2013) and 88 suggestions were made to improve the standard LRFD approach with refined partial factors 89 (e.g., Phoon et al. 2003b; Ching and Phoon 2011; Ching et al. 2013). In this paper, however, a 
90 different approach is taken and a new way to apply the existing LRFD codes with explicit 91 consideration of robustness is proposed.

92 In general, when applying a LRFD code for the design of a geotechnical structure, the 93 nominal values of uncertain input parameters (e.g., resistance term and load term) should be 94 selected based on the knowledge of how the partial factors are calibrated. In the absence of this 95 knowledge, a conservative estimate may be used in practice based on an evaluation of the mean 96 as well as the level of variation (e.g., Orr 2000; Driscoll and Simpson 2001), or based on local 97 experience and engineering judgment. However, the level of variation of a soil parameter often 98 cannot be quantified with certainty due to the spatial variability and/or limited data availability 99 (Juang et al. 2013). Thus, the selection of the nominal values of soil parameters that are 100 required in the existing LRFD approach is quite uncertain or subjective. Given the uncertain 101 nature in the selection of the nominal values of uncertain parameters, the predicted performance 102 of a design based on the LRFD analysis would be uncertain. Facing the uncertainty in the 103 predicted performance, a conservative design is preferred. However, an overly conservative 104 design is cost-inefficient and it diminishes the advantage of LRFD, which is supposed to 105 perform better than the FS-based design approach in terms of producing more consistent design. 106 Therefore, even with LRFD, the need to reduce the potential uncertainty in the predicted 107 performance is still apparent.

108 To reduce the uncertainty in the predicted performance of a given geotechnical design 109 in the face of above variations (i.e., the uncertainties in soil parameters, solution model, applied 110 loads, and those caused by construction), a new design philosophy was advanced known as 111 robust geotechnical design (RGD) (Juang et al. 2013). Note that robust design concept has long 112 been utilized in engineering fields such as industrial, mechanical, structural, and aeronautical 
113 engineering (e.g., Doltsinis et al. 2005; Zhang et al. 2005; Park et al. 2006; Lagaros and 114 Fragiadakis 2007; Marano et al. 2008; Lee et al. 2010). The purpose of robust design is to 115 derive a design that effectively accounts for the effect of the variation in "noise factors" 116 (Taguchi 1986) while simultaneously considers the safety and cost efficiency. The term noise 117 factor that is typically used in the literature of robust design is referred to herein as the input 118 parameter that is hard-to-control (i.e., cannot be easily adjusted by the designer) and hard-to119 characterize (i.e., the uncertainty is recognized but difficult to quantify).

120 In the existing RGD approach, the safety is often evaluated with the failure probability 121 or reliability index; thus, the reliability analysis of the system response is an integral part of 122 RGD. Note that in this paper the term "system response" is referred to, within the context of 123 robust design, as the response of a structure or a component, or simply as the output of a model. 124 This term is not used in the context of system reliability, where multiple failure modes are 125 involved. Although theoretically sound, the implementation of the existing reliability-based 126 RGD approach (Juang et al. 2013) poses a significant challenge to the practicing engineer, 127 since the tasks of the statistical characterization of noise factors and the reliability analysis of 128 the system response must be performed within the robust design optimization framework. In 129 this paper, we introduce a modified RGD approach that shares the familiar look-and-feel and 130 convenience of the standard LRFD approach. This modified RGD approach is referred to 131 herein as Robust Load and Resistance Factor Design ( $R$-LRFD).

132 The $R$-LRFD is a new and simpler variant of reliability-based RGD, just like LRFD (if 133 it is calibrated through reliability analysis) is a simpler variant of RBD. It is an important step 134 forward to make RGD more practical, because $R$-LRFD does not require engineers to perform 
135 statistical characterization of noise factors and reliability analysis of the system response (same 136 advantages that LRFD has over RBD). Other significant features of the $R$-LRFD include:

137

138

147

148

150

153

154

155

156

1) A modified gradient-based robustness measure is proposed, with which the robustness of a system with recognized but unquantified uncertainties can be evaluated. This new robustness measure is suitable for integration with LRFD.

2) A new simplified procedure to identify the most preferred design in the design space is developed. With this new procedure, the optimal design with respect to multiple objectives can easily be identified through a series of single objective optimizations. This reduces the computation effort for robust design optimization and eliminates the need for the more complex multi-objective optimization algorithms.

The rest of this paper is organized as follows. First, the standard LRFD approach is briefly described to set the stage. Second, an updated gradient-based robustness measure, in terms of the sensitivity index (SI), is formulated. Third, the optimization framework of the proposed $R$-LRFD approach is presented. Fourth, the proposed $R$-LRFD approach is applied to two geotechnical problems: the design of drilled shaft in sand (illustrated with a discrete design space) and the design of drilled shaft in clay (illustrated with a continuous design space). They are followed with concluding remarks.

\section{Load and Resistance Factor Design (LRFD)}

Given a geotechnical structure with design parameters d (e.g., geometries) and uncertain parameters $\boldsymbol{\theta}$ (e.g., soil parameters, model errors, applied loads, and construction 
157 variation) as inputs, the system response, denoted as $f(\mathbf{d}, \boldsymbol{\theta})$, is a function of $\mathbf{d}$ and $\boldsymbol{\theta}$, expressed 158 as:

$$
f(\mathbf{d}, \boldsymbol{\theta})=R(\mathbf{d}, \boldsymbol{\theta})-S(\mathbf{d}, \boldsymbol{\theta})
$$

160 where $R(\mathbf{d}, \boldsymbol{\theta})$ and $S(\mathbf{d}, \boldsymbol{\theta})$ are the resistance term and the load term, respectively. Generally, the 161 system response function formulated in Eq. (1) is set up using an analytical or empirical model 162 or through a numerical method. To account for the effect of uncertain parameters $(\boldsymbol{\theta})$ on the 163 predicted performance (and thus the final design outcome), the safety, in the context of the 164 standard LRFD approach, is evaluated as:

$$
g\left(\mathbf{d}, \boldsymbol{\theta}^{\mathbf{n}}\right)=\psi_{R} R\left(\mathbf{d}, \boldsymbol{\theta}^{\mathbf{n}}\right)-\psi_{S} S\left(\mathbf{d}, \boldsymbol{\theta}^{\mathbf{n}}\right)
$$

166 where $R\left(\mathbf{d}, \theta^{\mathbf{n}}\right)$ and $S\left(\mathbf{d}, \theta^{\mathbf{n}}\right)$ are the nominal values of the resistance term and the load term,

167 respectively; $\boldsymbol{\theta}^{\mathbf{n}}$ are the nominal values of uncertain parameters $(\boldsymbol{\theta})$; and, $\psi_{R}$ and $\psi_{S}$ are the 168 resistance factor and the load factor, respectively. Note that the nominal values of the resistance 169 term and the load term are defined in this paper as the functions of the nominal values of 170 uncertain input parameters, although the nominal values of the resistance term and the load 171 term are typically estimated directly in LRFD. The reason is that this paper seeks to show that $172 R$-LRFD solutions are insensitive to the uncertain parameters $(\boldsymbol{\theta})$. It is recognized that the 173 application of $\boldsymbol{\theta}^{\mathbf{n}}$ in design is comparable to the characteristic value approach in Eurocode 7 , 174 where $\theta^{\mathbf{n}}$ could be defined at $5 \%$ for stabilizing parameters. In general, the resistance factor is 175 less than 1.0 and the load factor is greater than 1.0. The nominal values $\left(\boldsymbol{\theta}^{\mathbf{n}}\right)$ may be selected as 176 the mean or some percentile (e.g., 5\% for stabilizing parameter and $95 \%$ for destabilizing 177 parameter) of uncertain parameters $(\boldsymbol{\theta})$. It is noted that in the scenario involving multiple load 
or resistance terms, the system response function $f(\mathbf{d}, \boldsymbol{\theta})$ and LRFD performance function $g(\mathbf{d}$,

$\left.179 \theta^{\mathrm{n}}\right)$, shown in Eq. (1) and Eq. (2), respectively, should be modified as follows.

$$
f(\mathbf{d}, \boldsymbol{\theta})=\sum_{i} R_{i}(\mathbf{d}, \boldsymbol{\theta})-\sum_{j} S_{j}(\mathbf{d}, \boldsymbol{\theta})
$$

$$
g\left(\mathbf{d}, \boldsymbol{\theta}^{\mathbf{n}}\right)=\sum_{i} \psi_{R i} R_{i}\left(\mathbf{d}, \boldsymbol{\theta}^{\mathbf{n}}\right)-\sum_{j} \psi_{S j} S_{j}\left(\mathbf{d}, \boldsymbol{\theta}^{\mathbf{n}}\right)
$$

182

where $R_{i}(\mathbf{d}, \boldsymbol{\theta})$ and $S_{j}(\mathbf{d}, \boldsymbol{\theta})$ are the $i^{\text {th }}$ resistance term and the $j^{\text {th }}$ load term, respectively; $R_{i}\left(\mathbf{d}, \theta^{\mathbf{n}}\right)$ and $S_{j}\left(\mathbf{d}, \theta^{\mathbf{n}}\right)$ are the nominal values of the $i^{\text {th }}$ resistance term and the $j^{\text {th }}$ load term, respectively; and, $\psi_{R i}$ and $\psi_{S j}$ are the corresponding resistance factor and load factor, respectively.

For a candidate design $(\mathbf{d})$, if $g\left(\mathbf{d}, \boldsymbol{\theta}^{\mathbf{n}}\right)>0$, the safety requirement is satisfied; otherwise, the design is considered infeasible or unsafe. The uncertainties in the environment, whether they can be characterized probabilistically or by other means, or they are hard-to-characterize noises, have been taken care of through a combination of the nominal values and LRFD partial factors. For illustration purposes, the LRFD partial factors are applied to the nominal values of the resistance term and the load term in Eq. (2). Alternatively, the LRFD partial factors can be applied to the nominal values of uncertain input parameters; however, these two different ways of applying the LRFD partial factors lead to similar results.

The LRFD partial factors (e.g., $\psi_{R}$ and $\psi_{S}$ ) are constants that have been calibrated for the design of a geotechnical structure using a given solution model at a target reliability index. However, the selection of the nominal values for uncertain parameter $\left(\theta^{\mathbf{n}}\right)$ (and hence the nominal values of the load and resistance terms) is not straightforward. The nominal values of uncertain parameters at a given site under a given design scenario can be rather uncertain, although the standard LRFD approach treats them as constants. Given the recognized but 
unquantified uncertainty associated with the nominal values, the potential risk of the design

200 obtained with the existing LRFD code is unknown. Thus, the existing LRFD code has the same 201 dilemma as one facing the traditional FS-based design approach: it may produce an inefficient 202 over-design with an unknown and/or inconsistent safety level, although under-design is also a 203 possibility. In fact, for LRFD codes that are not calibrated using rigorous reliability methods, 204 there is no rational basis to assess if the design outcome is conservative or vice-versa. This is a 205 fundamental limitation faced by all design approaches that rely solely on judgment and 206 experience, though they enjoy the practical advantage of not having to quantify uncertainty. 207 The proposed $R$-LRFD approach retains the advantage of experience-based design approaches 208 while attempting to define a rational basis for optimal design, under the assumption that the 209 data are insufficient for reliability analysis.

210 By introducing the robust design concept into the standard LRFD approach, the effect 211 of the recognized but unquantified uncertainty in the nominal values of uncertain parameters on 212 the final design can be explicitly dealt with. The purpose of robust design is to seek an optimal 213 design such that the system response is robust against the uncertainty in noise factors (in this 214 case, the uncertainty in the nominal values). Note that the uncertainty in the nominal values of 215 uncertain parameters may be attributed to either the absence of the knowledge of how the 216 partial factors are derived or the uncertainty in quantifying the level of variation of uncertain 217 parameters. To integrate the robust design concept into the standard LRFD approach, the 218 recently developed gradient-based robustness measure, in terms of the sensitivity index (SI) 219 (Gong et al. 2014b), is modified herein. This modified gradient-based robustness measure does 220 not require statistical characterization of noise factors; however, the effect of the recognized but 
unquantified uncertainties in the noise factors can be explicitly considered; which is consistent with the spirit of the standard LRFD approach.

\section{Updated Gradient-Based Robustness Measure: Sensitivity Index (SI)}

In the context of robust design, the input parameters are classified into two categories, in terms of the design parameters $(\mathbf{d})$ and the noise factors $(\boldsymbol{\theta})$. The design parameters $(\mathbf{d})$ are those that can be easily adjusted by the designer, such as the geometries of the design; the noise factors $(\theta)$ are those that cannot be easily adjusted by the designer and that exhibit recognized but unquantified uncertainty, such as the soil parameters, model errors, applied loads, and construction variation. The purpose of robust design is to seek an optimal design, represented by a set of design parameters $(\mathbf{d})$, in which the system response $[f(\mathbf{d}, \boldsymbol{\theta})]$ is robust against, or insensitive to, the variation in noise factors ( $\boldsymbol{\theta})$ (Taguchi 1986).

To illustrate the concept of robust design, the response of a system with noise factors is

234 demonstrated in Figure 1. Figure 1(a) shows the large variation in the performance of design $\mathbf{d}_{\mathbf{1}}$ 235 as a result of the combination of the large variation in noise factors $\theta_{1}$ and the high sensitivity 236 (or low robustness) of the system response to the noise factors, which poses a challenge in the 237 design decision making. Figure 1(b) and 1(c) depict two approaches to reduce the variation in 238 the system response. Figure 1(b) achieves this goal by focusing on reducing the variation in 239 noise factors (e.g., $\boldsymbol{\theta}_{2}$ in Figure $1 \mathrm{~b}$ versus $\boldsymbol{\theta}_{\mathbf{1}}$ in Figure $1 \mathrm{a}$ ), which may be realized by adopting a 240 more comprehensive site characterization program and/or use of a more accurate and precise 241 solution model. Figure 1(c) achieves the same goal with robust design that optimizes the design 242 parameters without reducing the variation in noise factors, (e.g., $\mathbf{d}_{\mathbf{2}}$ in Figure $1 \mathrm{c}$ versus $\mathbf{d}_{\mathbf{1}}$ in 
243 Figure 1a). The latter is realized by minimizing the sensitivity, or maximizing the robustness,

244 of the system response to the noise factors.

245 It is noted that the reduction of the variation in noise factors $(\theta)$ is not the focus of this

246 paper; rather, the focus is placed on the optimization of design parameters (d) through robust

247 design in the face of the recognized but unquantified uncertainty in geotechnical engineering.

248 The key to the success of the robust design is the measure of robustness. To set the stage for the

249 development of a robustness measure for a system with recognized but unquantified uncertainty,

250 the robustness measures for a system with uncertain parameters that have been statistically

251 quantified, as in the reliability-based robust design approach (Juang et al. 2013), are reviewed.

$252 \quad 3.1$ Robustness measure for a system with quantified uncertain parameters

253 For a design $(\mathbf{d})$ with uncertain input parameters $(\boldsymbol{\theta})$ that have been fully characterized

254 statistically, the robustness of the system response $[f(\mathbf{d}, \boldsymbol{\theta})]$, expressed in terms of the variability

255 of the system response, can be studied with probabilistic methods. For example, the standard

256 deviation of the system response, denoted as $\sigma_{f}$, may be obtained using the mean value first

257 order second moment method (MFOSM) (Ang and Tang 2007).

258

$$
\sigma_{f} \approx \sqrt{\left(\left.\mathbf{G}\right|_{\theta=\mu_{\theta}}\right) \cdot \mathbf{C}_{\theta} \cdot\left(\left.\mathbf{G}\right|_{\theta=\mu_{\theta}}\right)^{\mathrm{T}}}
$$

259 where $\mathbf{C}_{\boldsymbol{\theta}}$ is the covariance matrix among the uncertain parameters $(\boldsymbol{\theta})$, and $\left.\mathbf{G}\right|_{\theta=\mu_{\theta}}$ is the

260 gradient of the system response $[f(\mathbf{d}, \boldsymbol{\theta})]$ to the uncertain parameters $(\boldsymbol{\theta})$ evaluated at the mean

261 of uncertain parameters $\left(\mu_{\theta}\right)$, which is formulated as (Gong et al. 2014b):

262

$$
\left.\mathbf{G}\right|_{\theta=\mu_{\theta}}=\left\{\left.\frac{\partial f(\mathbf{d}, \boldsymbol{\theta})}{\partial \theta_{1}}\right|_{\theta=\mu_{\theta}},\left.\frac{\partial f(\mathbf{d}, \boldsymbol{\theta})}{\partial \theta_{2}}\right|_{\theta=\mu_{\theta}}, \cdots,\left.\frac{\partial f(\mathbf{d}, \boldsymbol{\theta})}{\partial \theta_{n}}\right|_{\theta=\mu_{\theta}}\right\}
$$


where $n$ is the number of uncertain parameters $(\theta)$.

Further, the mean of the system response $[f(\mathbf{d}, \boldsymbol{\theta})]$, denoted as $\mu_{f}$, can be approximated using MFOSM.

$$
\mu_{f} \approx f\left(\mathbf{d}, \boldsymbol{\mu}_{\theta}\right)=R\left(\mathbf{d}, \boldsymbol{\mu}_{\theta}\right)-S\left(\mathbf{d}, \boldsymbol{\mu}_{\theta}\right)
$$

Then, the variability, expressed in terms of the coefficient of variation (COV), of the system response $[f(\mathbf{d}, \boldsymbol{\theta})]$, denoted as $\delta_{f}$, can be evaluated as follows.

$$
\delta_{f}=\frac{\sigma_{f}}{\mu_{f}} \approx \frac{\sqrt{\left(\left.\mathbf{G}\right|_{\theta=\mu_{\theta}}\right) \cdot \mathbf{C}_{\theta} \cdot\left(\left.\mathbf{G}\right|_{\theta=\mu_{\theta}}\right)^{\mathrm{T}}}}{f\left(\mathbf{d}, \mu_{\theta}\right)}
$$

Intuitively, a lower variability of the system response $\left(\delta_{f}\right)$ signals a higher degree of robustness. For a geotechnical structure with uncertain parameters that have been characterized

272 statistically, the robustness may be formulated in various forms, such as feasibility robustness

273 (Du and Chen 2000; Juang et al. 2013) and standard deviation of the system response of 274 concern (Juang et al. 2014). As has been reported, these robustness measures are all consistent 275 with another popular robustness measure, namely, signal-to-noise-ratio (SNR) (Taguchi 1986;

276 Phadke 1989; Park et al. 2006; Gong et al. 2014a).

\subsection{Robustness measure for a system with unquantified uncertain parameters}

The traditional robustness measures that are based on probabilistic analyses, such as the coefficient of variation, signal-to-noise-ratio (SNR) and feasibility robustness, are suitable for

280 the system with quantified uncertain parameters. They are, however, not applicable to the 281 system with unquantified uncertain parameters (how do you proceed without the knowledge of 282 the statistics of uncertain parameters?). In other words, the existing robustness measures cannot 283 be integrated with the standard LRFD approach. 
The robustness or insensitivity of the system response $[f(\mathbf{d}, \boldsymbol{\theta})]$ to the variation in noise 285 factors $(\boldsymbol{\theta})$ can be measured with its gradient $(\mathbf{G})$, which is formulated as the variation of the 286 system response caused by one unit change in noise factors (Gong et al. 2014b). The plots in 287 Figure 1(a) and 1(c) illustrate the gradient-based robustness concept: two designs (i.e., $\mathbf{d}_{\mathbf{1}}$ and $288 \mathbf{d}_{2}$ ) with the same noise factors $\left(\boldsymbol{\theta}_{\mathbf{1}}\right)$ exhibit different patterns of system response, one (i.e., $\mathbf{d}_{\mathbf{1}}$ in 289 Figure 1a) yields high variation in the system response and the other (i.e., $\mathbf{d}_{2}$ in Figure 1c) 290 yields low variation in the system response. The design that yields lower variation in the system 291 response, known as a more robust design by definition, is shown to have a lower gradient (G). 292 Thus, the gradient $(\mathbf{G})$ is an effective measure of robustness, and a robust design can be found 293 by lowering the gradient of the system response to the noise factors without quantifying the 294 uncertainties of noise factors. This unique characteristic of the gradient-based robustness 295 measure is a perfect match with LRFD, in which the uncertainties in input parameters and the 296 solution model are recognized but unquantified. Note that while the existing gradient-based 297 robustness measure (Gong et al. 2014b) is applicable for the scenario involving a system with 298 quantified uncertain parameters, the modified gradient-based robustness measure, outlined 299 below, is intended for the scenario involving a system with recognized but unquantified 300 uncertainty. The latter is uniquely suitable for integration with the standard LRFD.

301 Similar to Eq. (6), the gradient of the system response $[f(\mathbf{d}, \boldsymbol{\theta})]$ to the noise factors at a 302 checkpoint of $\boldsymbol{\theta}^{\prime}$, denoted as $\left.\mathbf{G}\right|_{\theta=\boldsymbol{\theta}^{\prime}}$, can be expressed as follows.

$$
\left.\mathbf{G}\right|_{\theta=\theta^{\prime}}=\left\{\left.\frac{\partial f(\mathbf{d}, \boldsymbol{\theta})}{\partial \theta_{1}}\right|_{\theta=\theta^{\prime}},\left.\frac{\partial f(\mathbf{d}, \boldsymbol{\theta})}{\partial \theta_{2}}\right|_{\theta=\theta^{\prime}}, \cdots,\left.\frac{\partial f(\mathbf{d}, \boldsymbol{\theta})}{\partial \theta_{n}}\right|_{\theta=\boldsymbol{\theta}^{\prime}}\right\}
$$


304 Within the framework of the standard LRFD approach using fixed nominal values of uncertain 305 parameters $\left(\boldsymbol{\theta}^{\mathbf{n}}\right)$ as inputs, it is intuitive to take these nominal values $\left(\boldsymbol{\theta}^{\mathbf{n}}\right)$ as the checkpoint of 306 noise factors $\left(\boldsymbol{\theta}^{\prime}\right)$, expressed as: $\boldsymbol{\theta}^{\prime}=\boldsymbol{\theta}^{\mathbf{n}}$. This formulation is consistent with the requirement of 307 using fixed nominal values in the standard LRFD. In addition, the $R$-LRFD framework is 308 predicated on unquantifiable uncertainties and it is thus compelled to minimize reliance on 309 statistics such as mean values. Although the gradient $\left(\left.\mathbf{G}\right|_{\theta=\theta^{\prime}}\right)$ in Eq. (9) is an effective indicator of robustness, the 311 following two problems need to be resolved before the gradient-based robustness measure can 312 be applied for the general robust design optimization. First, the gradient is a dimensional vector. 313 The units of noise factors are different, thus the optimization operation of the vector can be a 314 problem. Second, the gradient is a vector rather than a scalar, it is inconvenient to be used for 315 screening candidate designs in an optimization framework. That is to say, the gradient vector in 316 Eq. (9) must be normalized with scaling factors and then converted into a scalar for ease of 317 application in the robust design optimization. To overcome the first problem, each partial derivative of the gradient vector $\left(\left.\mathbf{G}\right|_{\theta=\theta^{\prime}}\right)$, in terms of $\left.\frac{\partial f(\mathbf{d}, \boldsymbol{\theta})}{\partial \theta_{i}}\right|_{\theta=\theta^{\prime}}$, is multiplied by a scaling factor of $w_{i} \frac{\theta_{i}^{\prime}}{f\left(\mathbf{d}, \boldsymbol{\theta}^{\prime}\right)}$ such that it becomes a 320 dimensionless parameter. Similar normalization methods can be found in Gonsamo (2011). The 321 gradient vector $\left(\left.\mathbf{G}\right|_{\theta=\theta^{\prime}}\right)$ in Eq. (9) is then re-written as a dimensionless vector $\left(\left.\mathbf{J}\right|_{\theta=\theta^{\prime}}\right)$.

$$
\left.\mathbf{J}\right|_{\theta=\theta^{\prime}}=\left\{\left.w_{1} \frac{\theta_{1}^{\prime}}{f\left(\mathbf{d}, \boldsymbol{\theta}^{\prime}\right)} \frac{\partial f(\mathbf{d}, \boldsymbol{\theta})}{\partial \theta_{1}}\right|_{\theta=\theta^{\prime}},\left.w_{2} \frac{\theta_{2}^{\prime}}{f\left(\mathbf{d}, \boldsymbol{\theta}^{\prime}\right)} \frac{\partial f(\mathbf{d}, \boldsymbol{\theta})}{\partial \theta_{2}}\right|_{\theta=\theta^{\prime}}, \cdots,\left.w_{n} \frac{\theta_{n}^{\prime}}{f\left(\mathbf{d}, \boldsymbol{\theta}^{\prime}\right)} \frac{\partial f(\mathbf{d}, \boldsymbol{\theta})}{\partial \theta_{n}}\right|_{\theta=\theta^{\prime}}\right\}
$$


324 where $w_{i}$ is the weighting factor of the $i^{\text {th }}$ noise factor $\left(\theta_{i}\right)$, which signals the contribution of the

$325 i^{\text {th }}$ noise factor $\left(\theta_{i}\right)$ to the robustness. For instance, a noise factor that exhibits higher variability 326 would contribute more to the robustness measure defined herein. Furthermore, the above 327 formulation is quite similar to the concept of elasticity in economics (Gillespie 2007).

328 To solve the second problem, the "length" of the scaled dimensionless vector ( $\left.\left.\mathbf{J}\right|_{\theta=\theta^{\prime}}\right)$ is 329 computed and defined herein as sensitivity index (SI).

$$
\mathrm{SI}=\sqrt{\left(\left.\mathbf{J}\right|_{\theta=\theta^{\prime}}\right) \cdot \mathbf{R}_{\boldsymbol{\theta}} \cdot\left(\left.\mathbf{J}\right|_{\theta=\theta^{\prime}}\right)^{\mathrm{T}}}
$$

331 where $\mathbf{R}_{\boldsymbol{\theta}}$ is a $n$ by $n$ matrix depicting the correlation structure among the noise factors $(\boldsymbol{\theta})$.

332 Note that the estimation of $\mathbf{R}_{\theta}$ does not require full statistical characterization (i.e., knowledge

333 of covariance $\mathbf{C}_{\theta}$ ). In the scenario that lacks the knowledge of $\mathbf{C}_{\boldsymbol{\theta}}$, as in the system with 334 recognized but unquantified uncertainties, $\mathbf{R}_{\boldsymbol{\theta}}$ may be estimated based on experience and 335 engineering judgment. As will be discussed later, a rough estimate of $\mathbf{R}_{\boldsymbol{\theta}}$ is sufficient to obtain 336 consistent design outcome. The sensitivity index (SI) offers a single value representation of the 337 robustness. A higher SI value signals a lower degree of robustness, as it suggests a greater level 338 of variation of the system response $[f(\mathbf{d}, \boldsymbol{\theta})]$.

339 It is noted that if the uncertainty of each noise factor could be quantified, the weighting 340 factor $w$ (Eq. 10) may be replaced by the COV of the noise factor and the nominal value $\left(\theta^{\mathrm{n}}\right)$ 341 may be replaced by the mean of the noise factor. Then, the sensitivity index (SI) defined in Eq. 342 (11) becomes the mean-centered first-order approximation of the COV of the system response $343\left(\delta_{f}\right)$. In such a scenario, this new robustness measure is seen as equivalent to the existing 344 robustness measures that based on probabilistic analyses. However, this new robustness 
345 measure is more useful under the pivotal assumption of unquantifiable noises. In the scenario

346 of unquantifiable noises, the proposed new robustness measure is a significant improvement

347 over the existing robustness measures. Finally, there is a question of whether the computed SI

348 (and thus the resulting design) is sensitive to the estimate of the weighting factors ( $w$ ) and the

349 correlation structure $\left(\mathbf{R}_{\boldsymbol{\theta}}\right)$. As is demonstrated later, the results of the robust design

350 optimizations are not sensitive to the estimate of the weighting factors $(w)$ and the correlation

$351 \quad$ structure $\left(\mathbf{R}_{\theta}\right)$.

352

\section{Robust Load and Resistance Factor Design (R-LRFD)}

A key benefit of the RGD approach is that it treats the geotechnical design as a multi-

355 objective optimization problem that considers safety, robustness, and cost efficiency

356 simultaneously (Juang et al. 2013; Gong et al. 2014a\&b). In this approach, the safety is a

357 compulsory constraint that must be satisfied, while the robustness and the cost efficiency are

358 the objectives to be optimized. A possible optimization setting of the proposed $R$-LRFD 359 approach is shown as follows:

Find: $\quad \mathbf{d}$ (design parameters)

Subject to: $\mathbf{d} \in \mathbf{D S}$ (design space) $g\left(\mathbf{d}, \boldsymbol{\theta}^{\mathbf{n}}\right)>0$ (safety)

Other constraints

Objectives: Minimize SI (a function of $f(\mathbf{d}, \boldsymbol{\theta})$ )

Minimize $C$ (cost)

361 where the constraint " $g\left(\mathbf{d}, \boldsymbol{\theta}^{\mathbf{n}}\right)>0$ " means "safe". In reference to Eq. (12), $R$-LRFD is to seek 362 an optimal design, represented by a set of design parameters (d), in the design space (DS) such 363 that the safety is satisfied, in terms of $g\left(\mathbf{d}, \boldsymbol{\theta}^{\mathbf{n}}\right)>0$, as in the standard LRFD approach, and the 
364 sensitivity index (SI) and the cost $(C)$ are minimized simultaneously. Note that the system

365 response function $f(\mathbf{d}, \boldsymbol{\theta})$, defined in Eq. (1), not $g\left(\mathbf{d}, \boldsymbol{\theta}^{\mathbf{n}}\right)$ defined in Eq. (2), is adopted for 366 evaluating the sensitivity index (SI) (Eqs. 9-11), which is an index for robustness in this paper; 367 this is the " $R$ " part of the proposed $R$-LRFD approach. Whereas, the system response function $368 g\left(\mathbf{d}, \theta^{\mathbf{n}}\right)$, defined in Eq. (2), is adopted for assessing the safety; this is the "LRFD" part of the $369 \quad$ proposed $R$-LRFD approach.

\section{$370 \quad 4.1$ Most preferred design based on the results of robust design optimization}

The objective to maximize the robustness (i.e., minimize the sensitivity index SI) and

372 the objective to minimize the cost are generally conflicting with each other. Thus, a design that 373 is optimal with respect to both objectives simultaneously is not attainable within the framework

374 of the proposed $R$-LRFD approach. Instead, the robust design optimization usually leads to a 375 set of non-dominated designs, which collectively defines a Pareto front that shows a tradeoff 376 between robustness and cost efficiency. The Pareto front might be obtained using genetic 377 algorithms such as Non-dominated Sorting Genetic Algorithm version II, NSGA-II (Deb et al. 378 2002; Juang et al. 2013). Alternatively, the Pareto front may be obtained through a series of 379 single-objective optimization procedures (Chen et al. 1999). Both approaches are employed in 380 this study; however, the focus is to develop a simplified procedure for obtaining the most 381 preferred design in the design space that can be integrated smoothly with $R$-LRFD without the 382 need to perform multi-objective optimization. The simplified procedure will be presented and 383 elaborated later.

384 By presenting a tradeoff between robustness and cost efficiency, the Pareto front can aid 385 in making an informed design decision. For example, either the least cost design that is above a 386 target robustness level or the most robust design that falls within a target cost level may be 
387 selected as the most preferred design in the design space. The determination of an appropriate 388 target level of robustness or cost, however, is problem-specific. When a strong preference is not 389 specified by the owner or client, the knee point on the Pareto front, which yields the best 390 compromise solution among the conflicting objectives, may be taken as the most preferred 391 design in the design space (Branke et al. 2004; Deb and Gupta 2011; Gong et al. 2014b). Once 392 the Pareto front is established, the knee point can be readily identified using the marginal utility 393 function approach (Branke et al. 2004; Gong et al. 2014b), normal boundary intersection 394 approach (Deb and Gupta 2011), or reflex angle approach (Branke et al. 2004; Deb and Gupta 395 2011). Figure 2(a) shows a conceptual illustration of the Pareto front and the knee point in a bi396 objective optimization problem that minimizes Objective 1 and Objective 2. In Figure 2(a), the 397 feasible domain is defined as a domain that consists of feasible or safe designs.

398 In most cases, the owner or client may only be interested in the final design or the most 399 preferred design in the design space, and not necessarily the whole Pareto front; further, the 400 designer would not like to perform multi-objective optimizations. In fact, if the most preferred 401 design in the design space can be identified directly, the sorting of Pareto front may not be 402 needed. To this end, a simplified procedure is developed herein to identify the most preferred 403 design in the design space. Coupling with this simplified procedure for the most preferred 404 design, the proposed $R$-LRFD approach has the potential as a practical design tool for the 405 general geotechnical engineering application.

406 The simplified procedure for identifying the most preferred design in the design space 407 that requires no multi-objective optimization is summarized as follows:

408 Step 1: Select a design space (DS) based on local experience and engineering judgment, 409 and screen for feasible (safe) designs based on the design constraints. 
Step 2: Conduct a single-objective optimization among all the feasible (safe) designs.

411 This step is repeated for each and every design objective. For example, in Figure 2(b), the most 412 optimal design with respect to Objective 1 and that with respect to Objective 2 are identified 413 separately.

414 Step 3: Identify a utopia design (in the space of the objective functions) for this multi415 objective optimization problem. Here, the utopia design is a "design" that is optimal with 416 respect to each and every design objective, as plotted in Figure 2(b). Note that this utopia 417 design is in reality not attainable if the objectives are conflicting with each other.

Step 4: Normalize the objective values of all feasible designs into a range of 0.0 to 1.0.

419 For example, the $i^{\text {th }}$ design objective, denoted as $b_{i}(\mathbf{d})$, can be normalized into $b_{i}^{\text {n }}(\mathbf{d})$ using the 420 following transformation.

$$
b_{i}^{\mathrm{n}}(\mathbf{d})=\frac{b_{i}(\mathbf{d})-\left[b_{i}(\mathbf{d})\right]_{\min }}{\left[b_{i}(\mathbf{d})\right]_{\max }-\left[b_{i}(\mathbf{d})\right]_{\min }}
$$

422 where $\left[b_{i}(\mathbf{d})\right]_{\max }$ and $\left[b_{i}(\mathbf{d})\right]_{\min }$ are the maximum and minimum objective values, respectively, 423 among all the optimal designs (obtained in Step 2) based on the $i^{\text {th }}$ design objective $\left[b_{i}(\mathbf{d})\right]$. This 424 normalization step is repeated for each and every design objective of concern.

Step 5: Calculate the distance from the utopia design to the candidate design (in the 426 feasible domain) in the normalized space of the objective functions. The design that yields the 427 minimum distance is taken as the most preferred design in the design space, as plotted in Figure $4282(b)$.

Theoretically, the computed distance from the utopia design to a candidate design may 430 be interpreted as the additional "price", in terms of a combination of robustness and cost 431 efficiency, the owner or client has to pay to select this candidate design as the final design. 
432 Although this idea can be traced back to the marginal utility concept (Branke et al. 2004) and 433 the compromise programming approach (Chen et al. 1999), the simplified procedure using the 434 "minimum distance" concept is a new contribution. As will be shown later, the most preferred 435 design in the design space obtained using this simplified procedure is practically the same as 436 the knee point on the Pareto front that is obtained based on the more comprehensive procedures 437 of multi-objective optimization. It is worth noting that the simplified procedure is applicable to 438 problems with more than two objectives and can be implemented with Microsoft Excel. If the 439 explicit system response function is not available, the response surface method (Zhang et al. $4402015)$ may be adopted to implement the proposed procedure with Excel.

\subsection{Procedures for implementing the proposed $R$-LRFD approach}

The procedures to implement the proposed $R$-LRFD approach are summarized in the 443 following main steps:

444 Step 1: Characterize the geotechnical problem of interest. In this step, the system 445 response of concern [i.e., $f(\mathbf{d}, \boldsymbol{\theta})$ and $g\left(\mathbf{d}, \boldsymbol{\theta}^{\mathbf{n}}\right)$ ], design parameters $(\mathbf{d})$, and noise factors $(\boldsymbol{\theta})$ are 446 identified, and the models for the safety, robustness, and cost are chosen. Further, the nominal 447 values of uncertain parameters $\left(\boldsymbol{\theta}^{\mathbf{n}}\right)$ in the standard LRFD approach are selected along with the 448 design space (DS). Finally, the weighting factors (w) and the correlation structure $\left(\mathbf{R}_{\theta}\right)$ are 449 estimated. For design of ordinary geotechnical structures, all weighting factors may be simply 450 set to 1.0 and the correlation structure set as an $n$-by- $n$ identity matrix.

Step 2: Evaluate the safety, in terms of $g\left(\mathbf{d}, \boldsymbol{\theta}^{\mathbf{n}}\right)>0$ (Eq. 2), robustness, in term of the 452 sensitivity index (SI) (Eqs. 9-11), and cost $(C)$ for each and every candidate design in the 453 design space (DS). 
Step 3: Obtain the most preferred design in the design space (DS) using the simplified procedure that is based on the minimum distance from the utopia design.

Step 4 (optional): Derive the Pareto front with the multi-objective optimization setting in Eq. (12). The most preferred design is then selected based on the preference of the owner or the knee point concept. This design may be used to verify the design obtained in Step 3.

\section{Illustrative Example 1 - Robust Design of a Drilled Shaft in Sand}

The first illustrative example considers the design of a drilled shaft in sand (Wang et al. design load of $800 \mathrm{kN}$ and the maximum settlement of this drilled shaft is required to be less than $25 \mathrm{~mm}$. Symbolically, they are expressed as: $F_{50}=800 \mathrm{kN}$ and $y_{a}=25 \mathrm{~mm}$, respectively.

\subsection{Geotechnical characterization for this drilled shaft problem}

Two fundamental requirements of concern in the design of drilled shafts are the bearing

467 capacity and the settlement. The former is referred to herein as the ultimate limit state (ULS) 468 requirement and the latter the serviceability limit state (SLS) requirement. For this drilled shaft 469 problem, the studies conducted by Wang et al. (2011) and Juang et al. (2013) found that the 470 design is controlled by the SLS requirement. Thus, only the SLS behavior of this drilled shaft is 471 considered herein and the corresponding system response function is formulated as follows.

$$
f(\mathbf{d}, \boldsymbol{\theta})=Q_{\mathrm{SLS}}-F_{\text {load }}
$$

473 where $Q_{\text {SLS }}$ is the SLS-based bearing capacity of the drilled shaft, detailed formulation of which 474 can be found in Appendix A; and, $F_{\text {load }}$ is the unfactored load acting on the drilled shaft while $475 \quad F_{50}$ is the factored load. 
In the context of the standard LRFD approach, the safety of the drilled shaft with 477 respect to the SLS requirement is assessed with the following equation (Phoon et al. 1995):

$$
g\left(\mathbf{d}, \boldsymbol{\theta}^{\mathbf{n}}\right)=\psi_{c} Q_{\mathrm{SLS}}-F_{50}
$$

where $\psi_{c}$ is the resistance factor, which is taken as 0.56 in this example (Phoon et al. 1995). If

$g\left(\mathbf{d}, \boldsymbol{\theta}^{\mathbf{n}}\right)>0$, the safety is satisfied; otherwise, the safety is not satisfied. In the standard LRFD approach, the resistance factor is adopted to consider the effect of the recognized but unquantified uncertainty in the perceived uncertain parameters (i.e., effective friction angle $\phi^{\prime}$ and coefficient of earth pressure at rest $K_{0}$ ).

In the proposed $R$-LRFD approach, the nominal values of uncertain parameters $\phi \prime$ and 485 $K_{0}$ are referred to herein as the noise factors $(\theta)$, while the dimensions of the drilled shaft (i.e., shaft diameter $B$ and shaft length $D$ ) are referred to herein as the design parameters (d). The cost of this drilled shaft $(C)$, in US dollars (USD), is expressed as a function of the design 488 parameters (d) as follows (R.S. Means Co. 2007; Wang et al. 2011).

$$
C(\mathbf{d})= \begin{cases}77.5 \times \frac{D}{0.3} & \text { for } B=0.9 \mathrm{~m} \\ 116.0 \times \frac{D}{0.3} & \text { for } B=1.2 \mathrm{~m} \\ 157.0 \times \frac{D}{0.3} & \text { for } B=1.5 \mathrm{~m}\end{cases}
$$

491 the mean values of $\phi^{\prime}$ and $K_{0}$ were estimated as $32^{\circ}$ and 1.0 , respectively; the COVs of $\phi^{\prime}$ and $492 K_{0}$ were estimated as $5 \%$ to $10 \%$ and $20 \%$ to $90 \%$, respectively; and, the correlation coefficient between $\phi^{\prime}$ and $K_{0}$ was estimated as -0.60 to -0.90 . To compare with the reliability-based RGD 
the correlation coefficient between $\phi^{\prime}$ and $K_{0}$ is not needed in the $R$-LRFD approach. However, to analyze the effect of the weighting factors and the correlation structure, which are included in the formulation of the sensitivity index, on the final design, the COVs of $7 \%$ and $50 \%$ for $\phi^{\prime}$ and $K_{0}$, respectively, and the correlation coefficient between $\phi^{\prime}$ and $K_{0}$ of -0.75 are assumed. other parameters, such as the unit weight of sand $\left(\gamma_{s}\right)$, unit weight of water $\left(\gamma_{w}\right)$, unit weight of concrete $\left(\gamma_{c}\right)$, ground water table $(H)$, and nominal in situ horizontal stress coefficient ratio

$503\left[\left(K / K_{0}\right)_{n}\right]$. These parameters are assumed to have fixed values as their variations are relatively 504 small and negligible. Here, they are assumed as: $\gamma_{s}=20 \mathrm{kN} / \mathrm{m}^{3}, \gamma_{w}=9.81 \mathrm{kN} / \mathrm{m}^{3}, \gamma_{c}=24 \mathrm{kN} / \mathrm{m}^{3}$, $505 H=0.0 \mathrm{~m}$, and $\left(K / K_{0}\right)_{n}=1.0$; these values are the same as those in Juang et al. (2013). Similar 506 to that in Juang et al. (2013), a discrete design space (DS) is used in this example: $\mathbf{D S}=\{(B$, $507 D) \mid B=\{0.9 \mathrm{~m}, 1.2 \mathrm{~m}, 1.5 \mathrm{~m}\}$ and $D=\{2.0 \mathrm{~m}, 2.2 \mathrm{~m}, 2.4 \mathrm{~m}, \ldots, 8.0 \mathrm{~m}\}\}$. In the selected design 508 space (DS), a total number of 93 discrete candidate designs are possible. Further, an additional 509 constraint of $D / B \geq 4$ is adopted such that the derived drilled shaft design is consistent with the 510 perception of deep foundation. Note that while a discrete design space is used in this example, a 511 continuous design space could also be handled, and similar results would be achieved, as is 512 demonstrated later in Example 2.

\section{$513 \quad 5.2$ Results of robust design of this drilled shaft problem}

514 The safety, robustness, and cost of each and every candidate design in the design space 515 (DS) are first evaluated. Then, the most preferred design in the design space (DS) of this drilled 516 shaft is identified using the simplified procedure based on the minimum distance concept 
517 described previously. The design parameters of the obtained most preferred design are: $B=0.9$

$518 \mathrm{~m}$ and $D=8.0 \mathrm{~m}$, and the corresponding cost and sensitivity index are: $C=2,067$ USD (raw 519 value) and $\mathrm{SI}=0.340$ (raw value), respectively. Listed in Table 1 are the detailed analysis 520 results of all these candidate designs. As can be seen in Table 1, the least cost design in the 521 feasible domain yields $B=0.9 \mathrm{~m}$ and $D=6.2 \mathrm{~m}$, which is associated with a sensitivity index of $522 \mathrm{SI}=0.482$ (raw value) and a cost of $C=1,602 \mathrm{USD}$ (raw value). On the other hand, the most 523 robust design in the feasible domain yields $B=1.5 \mathrm{~m}$ and $D=8.0 \mathrm{~m}$, which is associated with a 524 sensitivity index of $\mathrm{SI}=0.289$ (raw value) and a cost of $C=4,187$ USD (raw value).

Compared to the least cost design in the design space, the most preferred design yields a 526 significantly higher degree of robustness (normalized $\mathrm{SI}^{\mathrm{n}}=0.262$ versus 1.0 ) while the increase 527 in cost is only modest (normalized $C^{\mathrm{n}}=0.180$ versus 0.0 ). Compared to the most robust design, 528 the most preferred design yields significant savings in cost (normalized $C^{\mathrm{n}}=0.180$ versus 1.0 ) 529 while the robustness is lowered only slightly (normalized $\mathrm{SI}^{\mathrm{n}}=0.262$ versus 0.0 ). Therefore, 530 the design by $R$-LRFD (i.e., $B=0.9 \mathrm{~m}$ and $D=8.0 \mathrm{~m}$ ) yields the best compromise between cost 531 efficiency and robustness.

A Pareto front is further obtained by taking the optional step, which involves a multiobjective optimization algorithm (Deb et al. 2002). Figure 4 shows the derived Pareto front for 534 this drilled shaft problem, in both normalized objectives space (Figure 4a) and unnormalized 535 objectives space (Figure 4b). It is noted that the existence of three segments in this Pareto front, 536 as opposed to a single smooth curve, is caused by the adoption of a discrete design space (i.e., 537 with only three choices of shaft diameter $B$ ). The knee point on the Pareto front is identified 538 using the marginal utility function approach (Branke et al. 2004). The design parameters of the 539 derived knee point are: $B=0.9 \mathrm{~m}$ and $D=8.0 \mathrm{~m}$, which are the same as those of the most 
540 preferred design obtained using the simplified procedure. Thus, the optional step to obtain the

541 Pareto front is unnecessary if the intent of the designer is just to identify the most preferred

542 design in the design space.

\section{$543 \quad 5.3$ Effect of weighting factors and correlation structure on the final design}

544 A key component of the proposed $R$-LRFD approach is the updated robustness measure,

545 in terms of the modified sensitivity index (SI). According to Eqs. (9-11), this index appears to

546 depend upon the weighting factors $(w)$ and the correlation structure $\left(\mathbf{R}_{\theta}\right)$ of noise factors. The

547 question is whether the results of the proposed $R$-LRFD approach are affected by the estimate

548 of these parameters. To answer this question, let's recall the purpose of robust design, which is

549 to find the most preferred design in the design space. Is the contribution of the gradient alone,

550 as illustrated in Figure 1 and discussed in Eq. (9), which is represented by the scenario of equal

551 weights and no correlation, sufficient to constrain the search for the most preferred design? Or,

552 is the quantitative knowledge of the statistical characterization of noise factors crucial in

553 locating the most preferred design? A series of parametric analyses are performed herein to

554 investigate the effect of the assumption of the COVs (and thus weighting factors) and the

555 correlation structure on the outcome (i.e., the most preferred design) of the proposed $R$-LRFD

556 approach.

557 Tabulated in Table 2 are the $R$-LRFD outcomes obtained with different estimates of the

558 weighting factors (expressed in terms of the COV) and the correlation structure of noise factors.

559 It is found that the $R$-LRFD outcome is insensitive to the weighting factors and the correlation

560 structure of noise factors over a wide range of possibilities. Thus, the effect of the weighting

561 factors and the correlation structure on the final robust design is dominated by the influence of 562 the gradient term $(\mathbf{G})$. 
In addition to the results presented in Table 2, which are obtained based on limited 564 cases, 1,000 Monte Carlo simulations have been further conducted to generate the weighting 565 factors and the correlation structure. For these MCS runs, $w_{1}$ and $w_{2}$ are assumed uniformly 566 distributed over the possible ranges of $[5 \%, 10 \%]$ and $[20 \%, 90 \%]$, respectively; and, the 567 correlation coefficient between $\phi^{\prime}$ and $K_{0}$ is assumed uniformly distributed in the range of [-0.6, $568-0.9]$. In each of these MCS samples, the simplified procedure of the proposed $R$-LRFD 569 approach is applied and an optimal design is obtained. It is found that the variation of the final 570 designs among the 1000 simulations is relatively small and negligible. For example, the mean 571 of the resulting shaft diameter $(B)$ and shaft depth $(D)$ are $0.9 \mathrm{~m}$ and $8.0 \mathrm{~m}$, respectively; 572 whereas, the COV of the resulting $B$ and $D$ values are only $1.05 \%$ and $0.75 \%$, respectively. Further, given the statistical characterization of uncertain parameters in Table 2, this 574 drilled shaft is readily designed with RBD and reliability-based RGD (Gong et al. 2014b); and, 575 the derived designs are also included in Table 2. For illustration purposes, the target reliability 576 index $\left(\beta_{T}\right)$ is set to 2.6 and the reliability index $(\beta)$ of each candidate design is analyzed with 577 first order reliability method (FORM) (Low and Tang 2007). As can be seen in Table 2, the 578 outcomes obtained by RBD and reliability-based RGD are sensitive to the assumption of the 579 statistical information of uncertain parameters; whereas, the $R$-LRFD outcome is insensitive to 580 the assumption of the statistical information of uncertain parameters. The implication is that the 581 outcomes obtained by RBD and reliability-based RGD are meaningful only if the statistics of 582 uncertain parameters are accurately quantified; whereas, the proposed $R$-LRFD always yields a 583 consistent design in the scenario that involves the recognized but unquantified uncertainty. The 584 results shown in Table 2 also depict that the $R$-LRFD outcome and the reliability-based RGD 585 outcome are more conservative than the RBD outcome (indicated by a larger reliability index). 
586 In the case of $R$-LRFD, the conservativeness may be due to the adopted LRFD partial factors,

587 which are generally conservative. In the case of the reliability-based RGD, the conservativeness

588 is attributed to the consideration of design robustness, as reported by Gong et al. (2014b).

589 In summary, when applying the proposed $R$-LRFD approach, the most preferred design

590 can be obtained with a design scenario that sets all weighting factors to 1.0 and the correlation

591 structure as an $n$-by- $n$ identity matrix. Although this approach might be viewed as an

592 approximation, it yields a fairly accurate outcome (i.e., final design) and it is consistent with

593 the spirit of the standard LRFD approach that does not require a full statistical characterization

594 of uncertain parameters on the part of the user. Nevertheless, the formulations presented in Eqs.

595 (9-11) allow for an assessment and use of the statistical characterization of noise factors in the

596 evaluation of the robustness if so desired.

$597 \quad 5.4$ Effectiveness of the sensitivity index (SI) as a robustness measure

598 In the proposed $R$-LRFD approach, the sensitivity index (SI) of the system response to 599 the noise factors is used to measure the robustness. Here, the sensitivity index (SI) is compared 600 with the more traditional probabilistic analysis based robustness measures (i.e., standard 601 deviation of the failure probability $\sigma_{P_{f}}$ and feasibility robustness $\beta_{\beta}$ ), which were reported 602 previously in the reliability-based robust design of drilled shafts (Juang et al. 2013).

603 In order to calculate the more rigorous probability-based robustness measures, a full 604 statistical characterization of noise factors is needed. Given the full statistical characterization 605 of noise factors in Table 3 , the standard deviation of the failure probability $\left(\sigma_{P_{f}}\right)$ and the 606 feasibility robustness index $\left(\beta_{\beta}\right)$ can readily be computed for all the non-dominated designs 
607 (i.e., designs on the Pareto front) plotted in Figure 4. Comparisons of these robustness measures

608 with the new robustness measure (SI) are shown in Figure 5.

609 Detailed procedures for computing the standard deviation of the failure probability $\left(\sigma_{P_{f}}\right)$

610 due to the variation of the COVs are documented in Juang et al. (2013). The feasibility 611 robustness index $\left(\beta_{\beta}\right)$ is defined as follows (Juang et al. 2013):

$$
\beta_{\beta}=\Phi^{-1}\left[\operatorname{Pr}\left(P_{f}-P_{f T} \leq 0\right)\right]
$$

613 where $P_{f T}$ is the target failure probability, which is taken as 0.0047 in this example; the term $614 \operatorname{Pr}\left(P_{f}-P_{f T} \leq 0\right)$ is the probability that the computed $P_{f}$ is not greater than $P_{f T}$; and, $\Phi(\cdot)$ is the 615 cumulative distribution function of the standard normal variable. Note that the feasibility 616 robustness index measures the confidence level (or probability) that the safety constraint, in the 617 form of the target failure probability, can still be satisfied even with the uncertainty (variation) 618 in the characterized statistics of uncertain parameters. Figure 5(a) shows that the standard deviation of the failure probability $\left(\sigma_{P_{f}}\right)$ increases 620 with the normalized sensitivity index $\left(\mathrm{SI}^{\mathrm{n}}\right)$; that is to say, a design with a lower sensitivity 621 index (indicating a higher degree of robustness) generally exhibits less variation in the 622 computed failure probability. This is consistent with the concept of a robustly designed system. 623 Similarly, Figure 5(b) depicts that the computed feasibility robustness index $\left(\beta_{\beta}\right)$ decreases with 624 the normalized sensitivity index $\left(\mathrm{SI}^{\mathrm{n}}\right)$; that is to say, a design with a higher sensitivity index 625 (indicating a lower degree of robustness) yields a lower feasibility robustness index, indicating 626 a less robust design. This is, again, consistent with the concept of robust design. As both the 627 standard deviation of the failure probability and the feasibility robustness have been proven as 628 effective robustness measures (Juang et al 2013), the results shown in Figure 5 support the 
629 claim that the gradient-based sensitivity index (SI) is an effective robustness measure. The

630 latter, however, requires only a deterministic analysis and thus is much simpler and more

631 computationally efficient than the former. This deterministic nature makes the sensitivity index

632 (SI) an ideal robustness measure to be used in the proposed $R$-LRFD approach.

633

634 6. Illustrative Example 2- Robust Design of a Drilled Shaft in Clay

635 The second example considers the design of a drilled shaft in clay (ISSMGE 2009). As

636 shown in Figure 6, this drilled shaft is designed to support a dead load $\left(F_{\mathrm{d}}\right)$ and a live load $\left(F_{1}\right)$;

637 and, the maximum settlement of this drilled shaft is required to be less than $20 \mathrm{~mm}$, expressed

638 as: $y_{a}=20 \mathrm{~mm}$. One main difference between this example and Example 1 is that a continuous

639 design space is adopted instead of a discrete design space (Example 1).

$640 \quad 6.1$ Geotechnical characterization for this drilled shaft problem

641 For this drilled shaft in clay, the studies conducted by Orr et al. (2011) and

642 Khoshnevisan et al. (2015) found that critical factor that controls the design is the ULS

643 requirement. Thus, only the ULS behavior of this drilled shaft is considered and the

644 corresponding system response is evaluated as follows:

$645(\mathbf{d}, \boldsymbol{\theta})=Q_{\mathrm{s}}+Q_{\mathrm{b}}-F_{\mathrm{d}}-F_{1}$

646 where $Q_{\mathrm{s}}$ and $Q_{\mathrm{b}}$ are the side resistance and the base resistance of the drilled shaft, respectively.

647 These two terms can be determined as follows:

648

$Q_{\mathrm{s}}=\pi B \alpha \int_{0}^{D} c_{\mathrm{u} 1} d z$

649

$$
Q_{\mathrm{b}}=\frac{\pi}{4} B^{2} N_{\mathrm{c}} c_{\mathrm{u} 2}
$$


650 where $c_{\mathrm{u} 1}$ is the undrained shear strength along the shaft length $(\mathrm{kPa}) ; \alpha$ is an empirical 651 adhesion coefficient, taken as 0.5 (e.g., Meyerhof 1976); $z$ is the depth of clay stratum $(\mathrm{m}) ; N_{\mathrm{c}}$ 652 is a base baring capacity factor, taken as 9 (e.g., Meyerhof 1976); and, $c_{\mathrm{u} 2}$ is the undrained 653 shear strength at the shaft base $(\mathrm{kPa})$.

654 In the context of the standard LRFD approach, the safety of the drilled shaft with 655 respect to the ULS requirement is assessed with the following equation (AASHTO 2014):

$$
g\left(\mathbf{d}, \boldsymbol{\theta}^{\mathbf{n}}\right)=\psi_{Q_{\mathrm{s}}} Q_{s}+\psi_{Q_{\mathrm{b}}} Q_{\mathrm{b}}-\psi_{F_{\mathrm{d}}} F_{\mathrm{d}}-\psi_{F_{1}} F_{1}
$$

657 where $\psi_{Q_{\mathrm{S}}}$ and $\psi_{Q_{\mathrm{b}}}$ are the resistance factors with respect to the side resistance $\left(Q_{\mathrm{s}}\right)$ and the 658 base resistance $\left(Q_{\mathrm{b}}\right)$, respectively; and, $\psi_{F_{\mathrm{d}}}$ and $\psi_{F_{1}}$ are the load factors with respect to the 659 dead load $\left(F_{\mathrm{d}}\right)$ and the live load $\left(F_{1}\right)$, respectively. In this example, $\psi_{Q_{\mathrm{s}}}=0.45, \psi_{Q_{\mathrm{b}}}=0.4, \psi_{F_{\mathrm{d}}}$ $660=1.25$, and $\psi_{F_{1}}=1.75(\mathrm{AASHTO} 2014)$.

661 The nominal values of the undrained shear strength (i.e., $c_{\mathrm{u} 1}$ and $\left.c_{\mathrm{u} 2}\right)$, dead load $\left(F_{\mathrm{d}}\right)$, 662 and live load $\left(F_{1}\right)$ are referred to herein as the noise factors $(\boldsymbol{\theta})$. In Khoshnevisan et al. (2015), 663 the normalized undrained shear strength of the clay, denoted as $c_{\mathrm{u}}{ }^{\mathrm{n}}=c_{\mathrm{u}} / z$, is characterized as a 664 lognormal random variable with a mean of 13.04 and a standard deviation of 4.15 . Thus, the 665 nominal value of the normalized undrained shear strength $\left(c_{\mathrm{u}}{ }^{\mathrm{n}}\right)$ is taken as 13.04 for simplicity. 666 In ISSMGE (2009), the nominal values of the dead load $\left(F_{\mathrm{d}}\right)$ and the live load $\left(F_{1}\right)$ are specified 667 as $300 \mathrm{kN}$ and $150 \mathrm{kN}$, respectively; and, the COVs of the dead load and the live load are 668 assumed as 0.1 and 0.18 , respectively, based on the study by Zhang et al. (2011). Further, the 669 correlation structure among the normalized undrained shear strength $\left(c_{\mathrm{u}}{ }^{\mathrm{n}}\right)$, dead load $\left(F_{\mathrm{d}}\right)$, and 670 live load $\left(F_{1}\right)$ is assumed to be a 3 by 3 identity matrix. 
672 and shaft length $D$ ) are referred to herein as the design parameters (d). In this example, a

673 continuous design space (DS) is considered: $\mathbf{D S}=\{(B, D) \mid 0.3 \mathrm{~m} \leq B \leq 0.6 \mathrm{~m}$ and $10 \mathrm{~m} \leq D \leq$

$67425 \mathrm{~m}$ \}. For ease of illustration, the cost of this drilled shaft $(C)$ is expressed with the volume of

675 the concrete, expressed as:

676

$$
C(\mathbf{d})=\frac{\pi B^{2}}{4} D
$$

677

678

679

680

681

682

683

684

685

686

687

688

689

690

691

692

\subsection{Results of robust design of this drilled shaft problem}

Using the $R$-LRFD approach described previously, the final design, in terms of the most preferred design in the design space (DS), of this drilled shaft can easily be identified. The process involves three single-objective optimizations: one is to identify the least cost design (based on $\operatorname{cost} C$ ), another one is to identify the most robust design (based on sensitivity index SI), and the last one is to identify the most preferred design using the simplified procedure (based on the minimum distance concept). The design parameters of the final design outcome using the $R$-LRFD approach are: $B=0.37 \mathrm{~m}$ and $D=25.00 \mathrm{~m}$, and the corresponding cost and sensitivity index are: $C=2.66 \mathrm{~m}^{3}$ (raw value) and $\mathrm{SI}=0.383$ (raw value), respectively. This most preferred design is listed in Table 4 along with the least cost design and the most robust design obtained for this drill shaft problem.

As can be seen in Table 4, the least cost design in the feasible domain yields $B=0.30 \mathrm{~m}$ and $D=20.30 \mathrm{~m}$, which is associated with a sensitivity index of SI $=0.465$ (raw value) and a cost of $C=1.43 \mathrm{~m}^{3}$ (raw value). On the other hand, the most robust design in the feasible domain yields $B=0.60 \mathrm{~m}$ and $D=25.00 \mathrm{~m}$, which is associated with a sensitivity index of SI = 0.352 (raw value) and a cost of $C=7.07 \mathrm{~m}^{3}$ (raw value). Thus, compared to the least cost 
693 design in the design space, the most preferred design yields a significantly higher degree of 694 robustness (normalized $\mathrm{SI}^{\mathrm{n}}=0.273$ versus 1.0) while the increase in cost is only modest 695 (normalized $C^{\mathrm{n}}=0.217$ versus 0.0 ). Compared to the most robust design, the most preferred 696 design yields significant savings in cost (normalized $C^{\mathrm{n}}=0.217$ versus 1.0) while the 697 robustness is lowered only slightly (normalized $\mathrm{SI}^{\mathrm{n}}=0.273$ versus 0.0 ). Thus, the $R$-LRFD 698 design (i.e., $B=0.37 \mathrm{~m}$ and $D=25.00$ ) yields the best compromise between cost efficiency and 699 robustness.

A Pareto front is further obtained by taking the optional step, which involves a multi701 objective optimization algorithm (Deb et al. 2002). Figure 7 shows the derived Pareto front for 702 this drilled shaft problem, in both normalized objectives space (Figure 7a) and unnormalized 703 objectives space (Figure 7b). Since a continuous design space (DS) is adopted in this example, 704 the resulting Pareto front shown in Figure 7 is a single smooth curve. The knee point on the 705 Pareto front is identified using the marginal utility function approach (Branke et al. 2004). The 706 design parameters of the derived knee point are: $B=0.37 \mathrm{~m}$ and $D=25.00 \mathrm{~m}$, which are the 707 same as the most preferred design obtained using the simplified procedure of the $R$-LRFD 708 approach. Thus, the optional step to obtain the Pareto front is unnecessary if the intent of the 709 designer is just to identify the most preferred design in the design space.

710 Furthermore, Table 5 compares the computational efforts of the simplified procedure 711 with existing multi-objective optimization procedure. The computation time is recorded using 712 the same Windows 7® PC equipped with a 8.0 GB RAM and an Intel® Core ${ }^{\text {TM }}$ i7-3630QM 713 CPU running at $2.40 \mathrm{GHz}$. The RGD approach with the simplified procedure for knee point 714 reduces the computation time drastically.

\section{$715 \quad 6.3$ Effect of weighting factors on the final design}


Similar to Example 1, a parametric study is conducted herein to investigate the effect of

717 the assumption of the COVs on the outcome of the proposed $R$-LRFD approach. For this drilled

718 shaft problem, a design scenario is assumed with all weighting factors setting to 1.0 and the

719 correlation structure setting as a 3 by 3 identity matrix. The robust design using the $R$-LRFD

720 approach with this design scenario yields exactly the same results: $B=0.37 \mathrm{~m}$ and $D=25.00 \mathrm{~m}$.

721 The conclusion reached in Example 1 is confirmed in Example 2. The effect of the weighting

722 factors on the final design is dominated by the gradient.

$723 \quad 6.4$ Effectiveness of the sensitivity index (SI) as a robustness measure

724 Here, the sensitivity index (SI) is compared with another popular robustness measure, 725 namely, signal-to-noise ratio (SNR) (Phadke 1989). For this illustrative example, the SNR of a

726 design $\mathbf{d}$ is computed as follows (Gong et al. 2015):

$$
\operatorname{SNR}(\mathbf{d})=10 \log _{10}\left(\frac{\mu_{f}^{2}}{\sigma_{f}^{2}}\right)
$$

where $\mu_{f}$ and $\sigma_{f}$ are the mean and the standard deviation of the system response $[f(\mathbf{d}, \boldsymbol{\theta})]$, respectively, which can readily be estimated using MFOSM (see Eq. 5 and Eq. 7).

The plots in Figure 8 indicate that the SNR decreases with the normalized sensitivity 731 index $\left(\mathrm{SI}^{\mathrm{n}}\right)$; thus, a design with a lower sensitivity index (indicating a higher degree of 732 robustness) generally exhibits a higher SNR value. This is consistent with the concept of robust 733 design. Since the SNR is one of the most well accepted robustness measure in the literature

734 (Taguchi 1986; Phadke 1989; Park et al. 2006), the results shown in Figure 8 support the claim 735 that the updated gradient-based sensitivity index (SI) is an effective robustness measure. The 736 latter, however, requires only a deterministic analysis and thus is much simpler and more 737 computationally efficient than the former. 


\section{Further Discussion: Comparison between LRFD and $R$-LRFD}

The $R$-LRFD approach retains the simplicity in the design process that makes the LRFD

741 approach a preference (from usability standpoint) over the RBD, and yet it allows for a more

742 informed design decision based on a simultaneous consideration of the safety, robustness, and

743 cost efficiency. Since the $R$-LRFD approach can remove some of the shortcomings of the

744 standard LRFD approach, and it requires little additional effort, it appears to be a method of

745 choice. Figure 9 shows the optimal design (typically based on cost) obtained by LRFD along

746 with the most preferred design obtained by the $R$-LRFD. The design by LRFD happens to be

747 the least cost design on the Pareto front. This is easily understood, as the Pareto front consists

748 of all non-dominated designs that satisfy the requirements of LRFD. Note that the least cost

749 design on the Pareto front is found to have the lowest robustness (i.e., with the highest $\mathrm{SI}^{\mathrm{n}}$

750 value). The design (i.e., most preferred design) obtained by the $R$-LRFD yields the best

751 compromise between robustness (represented by $\mathrm{SI}^{\mathrm{n}}$ ) and cost efficiency (represented by $C^{\mathrm{n}}$ ).

752

\section{8. Summary and Conclusions}

754 This paper presents a new design approach, termed Robust Load and Resistance Factor 755 Design $(R$-LRFD). The proposed $R$-LRFD approach treats the geotechnical design as a multi756 objective optimization problem that considers explicitly the robustness, in addition to the safety 757 and the cost efficiency. A simplified version of $R$-LRFD is also developed, which does not 758 requires the use of multi-objective optimization algorithms. The following conclusions are 759 reached based on the results presented: 
1) The proposed $R$-LRFD approach is found effective and capable of producing a design

761 that yields the best compromise between robustness and cost efficiency in the design space.

762 This $R$-LRFD approach is seen as an effective integration of the standard LRFD approach with 763 the robust geotechnical design (RGD) concept. $R$-LRFD is applicable to all scenarios of data 764 availability, from one extreme that sufficient data is available to characterize noise factors 765 statistically to another that no data is available to quantify noise factors, although it is most 766 useful under the pivotal assumption of unquantifiable noises.

767 2) The developed sensitivity index (SI) is shown as an effective robustness measure. The 768 SI is found valid and suitable for the purpose of robust design regardless of data availability 769 (i.e., whether data is sufficient to quantify noise factors statistically). While the SI is formulated 770 in a deterministic manner, it is consistent with the existing robustness measures (e.g., standard 771 deviation of the failure probability, feasibility robustness, and signal-to-noise ratio SNR). A 772 design with a higher sensitivity index always yields a larger variation of the failure probability, 773 a lower feasibility robustness index, and a lower SNR value, all indicating a lower degree of 774 robustness.

7753 3) The multi-objective optimization of the proposed $R$-LRFD approach generally results 776 in a Pareto front that depicts the tradeoff between robustness and cost efficiency, which can aid 777 in making an informed design decision. Without a specified preference, the knee point on the 778 Pareto front that yields the best compromise between robustness and cost efficiency is usually 779 selected as the final design. A simplified version of the $R$-LRFD approach is developed, which 780 is found to be able to produce a final design identical to the knee point on the Pareto front. The 781 simplified version of the proposed $R$-LRFD approach, which does not require a multi-objective 
782 optimization, can be implemented in Microsoft Excel using the Solver feature. Thus, $R$-LRFD 783 has a potential as a practical geotechnical design approach.

\section{Acknowledgments}

This material is based on work supported in part by the National Science Foundation

787 under Grant Number CMMI-1200117 (Project titled "Transforming Robust Design Concept 788 into a Novel Geotechnical Design Tool"). Any opinions, findings, and conclusions or 789 recommendations expressed in this material are those of the authors and do not necessarily 790 reflect the views of the National Science Foundation. The first author wishes to acknowledge 791 the financial support provided by the Glenn Department of Civil Engineering, Clemson 792 University through the Aniket Shrikhande Graduate Fellowship. The authors also wish to thank 793 Dr. Jianye Ching for his input regarding LRFD.

\section{References}

797 AASHTO (American Association of State Highway and Transportation Officials). (2014). LRFD Bridge Design Specifications. 7th ed. AASHTO, Washington, DC.

Ang, A.H.S., and Tang, W.H. (2007). Probability Concepts in Engineering: Emphasis on Applications to Civil and Environmental Engineering. 2nd edn. Wiley, New York.

Baecher, G.B., and Christian, J.T. (2003). Reliability and Statistics in Geotechnical Engineering. John Wiley and Sons, New York. 
803 Branke, J., Deb, K., Dierolf, H., and Osswald, M. (2004). "Finding knees in multi-objective optimization." Parallel Problem Solving from Nature-PPSN VIII, Springer Berlin Heidelberg, 722-731.

806

BSI (British Standards Institution). (2007). National Annex to Eurocode 7: BS EN 1997-1-2004: Geotechnical Design - Part 1: General Rules. BSI, Milton Keynes, UK.

Casagrande, A. (1965). "The role of the 'calculated risk' in earthwork and foundation 809 engineering." Journal of the Soil Mechanics and Foundations Division, 91(4), 1-40.

Cao, Z., and Wang, Y. (2014). "Bayesian model comparison and characterization of undrained shear strength." Journal of Geotechnical and Geoenvironmental Engineering, 140(6), 04014018.

Chen, W., Wiecek, M. M., and Zhang, J. (1999). “Quality utility-A compromise programming approach to robust design.” Journal of Mechanical Design, 121(2), 179-187.

815 Ching, J., and Phoon, K. K. (2011). "A quantile-based approach for calibrating reliability-based partial factors." Structural Safety, 33(4), 275-285.

817 Ching, J., Phoon, K.K., Chen, J.R., and Park, J.H. (2013). "Robustness of constant load and resistance factor design factors for drilled shafts in multiple strata." Journal of Geotechnical and Geoenvironmental Engineering, 139(7), 1104-1114.

Christian, J.T. (2004). "Geotechnical engineering reliability: How well do we know what we are doing?" Journal of Geotechnical and Geoenvironmental Engineering, 130(10), 9851003.

Deb, K., and Gupta, S. (2011). "Understanding knee points in bicriteria problems and their 825 implications as preferred solution principles.” Engineering Optimization, 43(11), 11751204. 
826 Deb, K., Pratap, A., Agarwal, S., and Meyarivan, T. (2002). "A fast and elitist multi-objective genetic algorithm: NSGA-II.” IEEE Transactions on Evolutionary Computation, 6(2), 182-197.

829 Doltsinis, I., Kang, Z., and Cheng, G. (2005). "Robust design of non-linear structures using 830 optimization methods." Computer Methods in Applied Mechanics and Engineering, $831 \quad$ 194(12), 1779-1795.

832 Du, X., and Chen, W. (2000). "Towards a better understanding of modeling feasibility 833 robustness in engineering design.” Journal of Mechanical Design, 122(4), 385-394.

834 Driscoll, R., and Simpson, B. (2001). EN1997 Eurocode 7: Geotechnical Design, Proceedings 835 of ICE-Civil Engineering, 144(6), 49-54.

836 Fenton, G.A., and Griffiths, D.V. (2008). Risk Assessment in Geotechnical Engineering, Wiley, 837 New York. ISBN: 978-0-470-17820-1.

838 Gillespie, A. (2007). Foundations of Economics. Oxford University Press, New York.

839 Gong, W., Wang, L., Juang, C.H., Zhang, J., and Huang, H. (2014a). "Robust geotechnical 840 design of shield-driven tunnels." Computers and Geotechnics, 56, 191-201.

841 Gong, W., Khoshnevisan, S., and Juang, C.H. (2014b). "Gradient-based design robustness 842 measure for robust geotechnical design.” Canadian Geotechnical Journal, 51(11), 1331$843 \quad 1342$.

844 Gong, W., Wang, L., Khoshnevisan, S., Juang, C.H., Huang, H., and Zhang, J. (2015). "Robust 845 geotechnical design of earth slopes using fuzzy sets." Journal of Geotechnical and $846 \quad$ Geoenvironmental Engineering, 141(1), 04014084. 
Gonsamo, A. (2011). "Normalized sensitivity measures for leaf area index estimation using three-band spectral vegetation indices.” International Journal of Remote Sensing, 32(7): 2069-2080.

Honjo, Y., and Amatya, S. (2005). "Partial factors calibration based on reliability analyses for square footings on granular soils." Géotechnique, 55(6), 479-491.

852

Honjo, Y., Suzuki, M., and Matsuo, M. (2000). "Reliability analysis of shallow foundations in reference to design codes development." Computers and Geotechnics, 26(3), 331-346.

Honjo, Y., Suzuki, M., Shirato, M., and Fukui, J. (2002). "Determination of partial factors for a vertically loaded pile based on reliability analysis." Soils and Foundations, 42(5), 91-109.

International Society of Soil Mechanics and Geotechnical Engineering (ISSMGE). (2009). “Design examples 2.” Evaluation of Eurocode 7, http://www.eurocode7.com/etc10/.

Juang, C. H., Wang, L., Liu, Z., Ravichandran, N., Huang, H., and Zhang, J. (2013). "Robust geotechnical design of drilled shafts in sand: New design perspective." Journal of Geotechnical and Geoenvironmental Engineering, 139(12), 2007-2019.

Juang, C.H., Wang, L., Hsieh, H. S., and Atamturktur, S. (2014). "Robust geotechnical design of braced excavations in clays." Structural Safety, 49, 37-44.

Khoshnevisan, S., Gong, W., Juang, C. H., and Atamturktur, S. (2015). "Efficient robust geotechnical design of drilled shafts in clay using a spreadsheet.” Journal of Geotechnical and Geoenvironmental Engineering, 141(2), 04014092.

Kulhawy, F.H. (1991). “Drilled shaft foundations.” Foundation Engineering Handbook, H. Y. Fang, ed., 2nd Ed., Van Nostrand Reinhold, New York, 537-552.

Lagaros, N.D., and Fragiadakis, M. (2007). "Fragility assessment of steel frames using neural networks.” Earthquake Spectra, 23(4), 735-752. 
870 Lee, M.C., Kelly, D.W., Degenhardt, R., and Thomson, R.S. (2010). "A study on the 871 robustness of two stiffened composite fuselage panels." Composite Structures, 92(2), 223$872 \quad 232$.

873 Low, B.K. (2005), "Reliability-based design applied to retaining walls." Géotechnique, 55(1), $874 \quad 63-75$.

875 Low, B.K., and Tang, W.H. (2007). "Efficient spreadsheet algorithm for first-order reliability 876 method.” Journal of engineering mechanics, 133(12), 1378-1387.

877 Low, B.K., and Phoon, K.K. (2015). "Reliability-based design and its complementary role to $878 \quad$ Eurocode 7 design approach.” Computers and Geotechnics, 65, 30-44.

879 Marano, G.C., Sgobba, S., Greco, R., and Mezzina, M. (2008). "Robust optimum design of 880 tuned mass dampers devices in random vibrations mitigation." Journal of Sound and $881 \quad$ Vibration, 313(3), 472-492.

882 Meyerhof, G.G. (1976). "Bearing capacity and settlement of pile foundations (11th Terzaghi 883 612 Lecture).” Journal of the Geotechnical Engineering, ASCE, 102(3), 195-228.

884 Najjar, S.S., and Gilbert, R.B. (2009). "Importance of lower-bound capacities in the design of 885 deep foundations.” Journal of Geotechnical and Geoenvironmental Engineering, 135(7), $886 \quad 890-900$.

887 NEN (Netherlands Standardization Institute). (2007). Eurocode 7: National Annex to NEN-EN 888 1997-1-2005: Geotechnical Design - Part 1: General Rules. NEN, Delft, Netherlands. 889 NSAI (National Standards Authority Ireland). (2005). National Annex Eurocode 7: I. S. EN 890 1997-1-2005: Geotechnical Design - Part 1: General Rules. NSAI, Dublin, Ireland.

891 Orr, T.L.L. (2000). "Selection of characteristic values and partial factors in geotechnical 892 designs to Eurocode 7.” Computers and Geotechnics, 26, 263-279. 
Orr, T.L.L., Bond, A.J., and Scarpelli, G. (2011). "Findings from the 2nd set of Eurocode 7 design examples.” Proc., 3rd Int. Symp. On Geotechnical Safety and Risk (ISGSR 2011), N. Vogt, B. Schuppener, D. Straub, and G. Braeu, eds., Bundesanstalt für Wasserbau, Munich, Germany, 537-547.

Paikowsky, S.G., Birgisson, B., Mcvay, M., and Nguyen, T. (2004). Load and Resistance Factor Design (LRFD) for Deep Foundations (No. 507). Transportation Research Board, Washington, D.C.

Park, G.J., Lee, T.H., Lee, K.H., and Hwang, K.H. (2006). "Robust design: an overview." AIAA Journal, 44(1), 181-191.

Park, J.H., Kim, D., and Chung, C.K. (2012). "Implementation of Bayesian theory on LRFD of axially loaded driven piles." Computers and Geotechnics, 42, 73-80.

Park, J.H., Huh, J., Kim, K.J., Chung, M., Lee, J.H., Kim, D., and Kwak, K. (2013). "Resistance factors calibration and its application using static load test data for driven steel pipe piles.” KSCE Journal of Civil Engineering, 17(5), 929-938.

Phadke, M.S. 1989. Quality Engineering Using Robust Design. Prentice Hall, New Jersey.

Phoon, K.K., Kulhawy, F.H., and Grigoriu, M.D. (1995). "Reliability based design of foundations for transmission line structures.” Rep. TR-105000, Electric Power Research Institute, Palo Alto, CA.

Phoon, K.K., Kulhawy, F.H., and Grigoriu, M.D. (2003a). "Development of a reliability-based design framework for transmission line structure foundations." Journal of Geotechnical and Geoenvironmental Engineering, 129(9), 798-806. 
914 Phoon, K.K., Kulhawy, F.H., and Grigoriu, M.D. (2003b). "Multiple resistance factor design 915 for shallow transmission line structure foundations." Journal of Geotechnical and $916 \quad$ Geoenvironmental Engineering, 129(9), 807-818.

917 R.S. Means Co. (2007). R.S. Means Building Construction Cost Data, Kingston, MA.

918 Standards Australia. (2009). AS2159-2009: Piling - Design and Installation. Standards 919 Australia, Sydney, New South Wales, Australia.

920 Stuedlein, A.W., Neely, W.J., and Gurtowski, T.M. (2012). "Reliability-based design of 921 augered cast-in-place piles in granular soils." Journal of Geotechnical and 922 Geoenvironmental Engineering, 138(6), 709-717.

923 Taguchi, G. (1986). Introduction to Quality Engineering: Designing Quality into Products and 924 Processes. Quality Resources, White Plains, New York.

925 Wang, Y., Au, S.K., and Kulhawy, F.H. (2011). "Expanded reliability based design approach 926 for drilled shafts.” Journal of Geotechnical and Geoenvironmental Engineering, 137(2), 927 140-149.

928 Wang, Y., and Cao, Z. (2013). "Probabilistic characterization of Young's modulus of soil using 929 equivalent samples.” Engineering Geology, 159, 106-118.

930 Zhang, J., Zhang, L.M., and Tang, W.H. (2009). "Bayesian framework for characterizing 931 geotechnical model uncertainty." Journal of Geotechnical and Geoenvironmental 932 engineering. 135(7) 932-940.

933 Zhang, J., Tang, W.H., and Zhang, L.M. (2010). Efficient probabilistic back-analysis of slope 934 stability model parameters. Journal of Geotechnical and Geoenvironmental Engineering, $935 \quad 136(1), 99-109$. 
936 Zhang, J., Zhang, L.M., and Tang, W.H. (2011). "Reliability-based optimization of 937 geotechnical systems." Journal of Geotechnical and Geoenvironmental Engineering, $938 \quad 137(12), 1211-1221$.

939 Zhang, J., Chen, H.Z., Huang, H.W., and Luo, Z. (2015). "Efficient response surface method 940 for practical geotechnical reliability analysis." Computers and Geotechnics, 69, 496-505.

941 Zhang, W., Chen, B.S., and Tseng, C.S. (2005). “Robust $\mathrm{H}_{\infty}$ filtering for nonlinear stochastic 942 systems.” Signal Processing, IEEE Transactions on, 53(2), 589-598. 


\section{List of Tables}

Table 1. Results of the robust design optimization for Example 1

Table 2. Final designs obtained by $R$-LRFD, RBD, and reliability-based RGD with different design scenarios of the statistical characterization of uncertain input parameters for Example 1

Table 3. A full statistical characterization of uncertain input parameters for Example 1

Table 4. Results of the robust design optimization for Example 2

Table 5. Comparison of the computational efficiency between the simplified procedure and the existing multi-objective optimization procedure 


\section{List of Figures}

Figure 1. Variation in the system response: (a) System response of a non-robust design $\mathbf{d}_{1}$ with noise factors as its input; (b) Reducing the variation in the system response by reducing the variation in noise factors; (c) Reducing the variation in the system response by adopting a robust design $\mathbf{d}_{\mathbf{2}}$ without reducing the variation in noise factors.

Figure 2. Conceptual illustration of a bi-objective optimization problem: (a) Pareto front and knee point; (b) Simplified procedure to identify the most preferred design in the design space

Figure 3. Schematic diagram of the design of a drilled shaft in sand (Example 1)

Figure 4. The Pareto front and knee point obtained for the drilled shaft in sand (Example 1): (a) Normalized design objectives; (b) Unnormalized design objectives

Figure 5. Effectiveness of the sensitivity index as a robustness measure (Example 1): (a) $\sigma_{P_{f}}$ versus $\mathrm{SI}^{\mathrm{n}}$; (b) $\beta_{\beta}$ versus $\mathrm{SI}^{\mathrm{n}}$

Figure 6. Schematic diagram of the design of a drilled shaft in clay (Example 2)

Figure 7. The Pareto front and knee point obtained for the drilled shaft in clay (Example 2): (a) Normalized design objectives; (b) Unnormalized design objectives

Figure 8. Effectiveness of the sensitivity index as a robustness measure (Example 2)

Figure 9. Comparison of designs obtained with LRFD and R-LRFD: (a) Example 1; (b) Example 2 
Table 1. Results of the robust design optimization for Example 1

\begin{tabular}{|c|c|c|c|c|c|c|c|c|c|}
\hline \multirow{2}{*}{ No. } & \multicolumn{2}{|c|}{$\begin{array}{c}\text { Design } \\
\text { parameters }\end{array}$} & \multirow{2}{*}{$\begin{array}{l}\text { Safety, } \\
g\left(\mathbf{d}, \boldsymbol{\theta}^{\mathbf{n}}\right)\end{array}$} & \multirow{2}{*}{$\begin{array}{l}\text { Cost, } \\
C\left(\mathrm{~m}^{3}\right)\end{array}$} & \multirow{2}{*}{$\begin{array}{l}\text { Sensitivity } \\
\text { index, } \\
\text { SI }\end{array}$} & \multirow{2}{*}{$\begin{array}{l}\text { Feasible } \\
\text { or safe? }\end{array}$} & \multicolumn{2}{|c|}{$\begin{array}{c}\text { Normalized } \\
\text { objectives }\end{array}$} & \multirow{2}{*}{$\begin{array}{c}\text { Computed } \\
\text { distance }\end{array}$} \\
\hline & $B(\mathrm{~m})$ & $\begin{array}{c}D \\
(\mathrm{~m})\end{array}$ & & & & & $C^{\mathrm{n}}$ & $\mathrm{SI}^{\mathrm{n}}$ & \\
\hline 1 & 0.9 & 2.0 & -561.64 & 516.67 & 0.309 & No & - & - & - \\
\hline 2 & 0.9 & 2.2 & -536.54 & 568.33 & 0.383 & No & - & - & - \\
\hline 3 & 0.9 & 2.4 & -511.15 & 620.00 & 0.482 & No & - & - & - \\
\hline 4 & 0.9 & 2.6 & -485.50 & 671.67 & 0.618 & No & - & - & - \\
\hline 5 & 0.9 & 2.8 & -459.58 & 723.33 & 0.821 & No & - & - & - \\
\hline \multicolumn{10}{|l|}{$\ldots$} \\
\hline 20 & 0.9 & 5.8 & -40.95 & 1498.33 & 0.543 & No & - & - & - \\
\hline 21 & 0.9 & 6.0 & -11.10 & 1550.00 & 0.510 & No & - & - & - \\
\hline $22^{(1)}$ & 0.9 & 6.2 & 19.00 & 1601.67 & 0.482 & Yes & 0.000 & 1.000 & 1.000 \\
\hline 23 & 0.9 & 6.4 & 49.34 & 1653.33 & 0.458 & Yes & 0.020 & 0.874 & 0.875 \\
\hline 24 & 0.9 & 6.6 & 79.92 & 1705.00 & 0.437 & Yes & 0.040 & 0.765 & 0.766 \\
\hline \multicolumn{10}{|l|}{$\ldots$} \\
\hline 29 & 0.9 & 7.6 & 236.42 & 1963.33 & 0.361 & Yes & 0.140 & 0.373 & 0.398 \\
\hline 30 & 0.9 & 7.8 & 268.45 & 2015.00 & 0.350 & Yes & 0.160 & 0.315 & 0.353 \\
\hline $31^{(2)}$ & 0.9 & 8.0 & 300.71 & 2066.67 & 0.340 & Yes & 0.180 & 0.262 & 0.318 \\
\hline 32 & 1.2 & 2.0 & -426.13 & 773.33 & 1.419 & No & - & - & - \\
\hline 33 & 1.2 & 2.2 & -387.68 & 850.67 & 3.217 & No & - & - & - \\
\hline \multicolumn{10}{|l|}{$\ldots$} \\
\hline 89 & 1.5 & 7.2 & 1259.29 & 3768.00 & 0.306 & Yes & 0.838 & 0.084 & 0.842 \\
\hline 90 & 1.5 & 7.4 & 1322.73 & 3872.67 & 0.301 & Yes & 0.879 & 0.061 & 0.881 \\
\hline 91 & 1.5 & 7.6 & 1386.49 & 3977.33 & 0.297 & Yes & 0.919 & 0.040 & 0.920 \\
\hline 92 & 1.5 & 7.8 & 1450.58 & 4082.00 & 0.293 & Yes & 0.960 & 0.019 & 0.960 \\
\hline $93^{(3)}$ & 1.5 & 8.0 & 1515.01 & 4186.67 & 0.289 & Yes & 1.000 & 0.000 & 1.000 \\
\hline
\end{tabular}

Note:

(1) The least cost design in the feasible domain.

(2) The most preferred design in the design space.

(3) The most robust design in the feasible domain. 
Table 2. Final designs obtained by $R$-LRFD, RBD, and reliability-based RGD with different design scenarios of the statistical characterization of uncertain input parameters for Example 1

\begin{tabular}{|c|c|c|c|c|c|c|c|c|c|c|c|c|}
\hline \multirow{3}{*}{$\begin{array}{c}\text { Design } \\
\text { scenario }\end{array}$} & \multicolumn{2}{|c|}{$\mathrm{COV}$} & \multirow{2}{*}{$\begin{array}{l}\text { Correlation } \\
\text { structure } \mathbf{R}_{\boldsymbol{\theta}}\end{array}$} & \multicolumn{3}{|c|}{$R$-LRFD } & \multicolumn{3}{|c|}{$\mathrm{RBD}$} & \multicolumn{3}{|c|}{$\begin{array}{c}\text { Reliability-based } \\
\text { RGD }\end{array}$} \\
\hline & $\phi^{\prime}$ & $K_{0}$ & & $\begin{array}{c}B \\
(\mathrm{~m})\end{array}$ & $\begin{array}{c}D \\
(\mathrm{~m})\end{array}$ & $\beta$ & $\begin{array}{c}B \\
(\mathrm{~m})\end{array}$ & $\begin{array}{c}D \\
(\mathrm{~m})\end{array}$ & $\beta$ & $\begin{array}{c}B \\
(\mathrm{~m})\end{array}$ & $\begin{array}{c}D \\
(\mathrm{~m})\end{array}$ & $\beta$ \\
\hline & $7 \%$ & $50 \%$ & {$\left[\begin{array}{cc}1.0 & -0.75 \\
-0.75 & 1.0\end{array}\right]$} & 0.9 & 8.0 & 6.29 & 0.9 & 5.6 & 2.65 & 0.9 & 8.0 & 6.29 \\
\hline $1^{(1)}$ & $5 \%$ & $30 \%$ & {$\left[\begin{array}{cc}1.0 & -0.60 \\
-0.60 & 1.0\end{array}\right]$} & 0.9 & 8.0 & 7.48 & 0.9 & 5.2 & 2.65 & 0.9 & 8.0 & 7.48 \\
\hline $2^{(1)}$ & $5 \%$ & $30 \%$ & {$\left[\begin{array}{cc}1.0 & -0.90 \\
-0.90 & 1.0\end{array}\right]$} & 0.9 & 8.0 & 13.60 & 0.9 & 5.0 & 2.85 & 0.9 & 7.6 & 12.22 \\
\hline $3^{(1)}$ & $5 \%$ & $70 \%$ & {$\left[\begin{array}{cc}1.0 & -0.60 \\
-0.60 & 1.0\end{array}\right]$} & 0.9 & 8.0 & 6.77 & 0.9 & 5.2 & 2.63 & 0.9 & 8.0 & 6.77 \\
\hline $4^{(1)}$ & $5 \%$ & $70 \%$ & {$\left[\begin{array}{cc}1.0 & -0.90 \\
-0.90 & 1.0\end{array}\right]$} & 0.9 & 7.8 & 12.30 & 0.9 & 4.8 & 3.10 & 0.9 & 7.4 & 11.24 \\
\hline $5^{(1)}$ & $10 \%$ & $30 \%$ & {$\left[\begin{array}{cc}1.0 & -0.60 \\
-0.60 & 1.0\end{array}\right]$} & 0.9 & 8.0 & 3.60 & 0.9 & 7.0 & 2.70 & 1.2 & 7.0 & 4.62 \\
\hline $6^{(1)}$ & $10 \%$ & $30 \%$ & {$\left[\begin{array}{cc}1.0 & -0.90 \\
-0.90 & 1.0\end{array}\right]$} & 0.9 & 8.0 & 5.36 & 0.9 & 6.6 & 2.87 & 1.2 & 6.6 & 5.86 \\
\hline $7^{(1)}$ & $10 \%$ & $70 \%$ & {$\left[\begin{array}{cc}1.0 & -0.60 \\
-0.60 & 1.0\end{array}\right]$} & 0.9 & 8.0 & 3.50 & 0.9 & 7.0 & 2.70 & 1.2 & 7.0 & 4.63 \\
\hline $8^{(1)}$ & $10 \%$ & $70 \%$ & {$\left[\begin{array}{cc}1.0 & -0.90 \\
-0.90 & 1.0\end{array}\right]$} & 0.9 & 8.0 & 6.27 & 0.9 & 6.0 & 2.79 & 1.2 & 6.2 & 6.59 \\
\hline $9^{(2)}$ & - & - & {$\left[\begin{array}{cc}1.0 & 0 \\
0 & 1.0\end{array}\right]$} & 0.9 & 8.0 & - & - & - & - & - & - & - \\
\hline
\end{tabular}

Note:

(1) For noise factors, the weighting factors are taken as the COVs of noise factors for $R$-LRFD.

(2) Equal weights and an identity correlation matrix are adopted for $R$-LRFD. 
Table 3. A full statistical characterization of uncertain input parameters for Example 1

\begin{tabular}{|c|c|c|c|c|c|}
\hline \multirow{2}{*}{$\begin{array}{l}\text { Soil } \\
\text { parameter }\end{array}$} & \multirow[b]{2}{*}{$\begin{array}{l}\text { Type of } \\
\text { distribution }\end{array}$} & \multirow[b]{2}{*}{ Mean } & \multicolumn{3}{|c|}{ Coefficient of variation (COV) } \\
\hline & & & $\begin{array}{l}\text { Approximate } \\
\text { range }\end{array}$ & $\begin{array}{c}\text { Mean of } \\
\text { COV }\end{array}$ & $\begin{array}{l}\text { Std. Dev. } \\
\text { of COV }\end{array}$ \\
\hline $\begin{array}{l}\text { Effective } \\
\text { friction } \\
\text { angle, } \phi\end{array}$ & Lognormal & $32^{\circ}$ & $5 \%$ to $10 \%$ & $7 \%$ & $1.25 \%$ \\
\hline $\begin{array}{l}\text { Coefficient } \\
\text { of earth } \\
\text { pressure at } \\
\text { rest, } K_{0}\end{array}$ & Lognormal & 1.0 & $20 \%$ to $90 \%$ & $50 \%$ & $17.50 \%$ \\
\hline
\end{tabular}

Note:

The correlation coefficient between $\phi^{\prime}$ and $K_{0}$ is assumed normally distributed with a mean of 0.75 and a standard deviation (Std. Dev.) of 0.075. 
Table 4. Results of the robust design optimization for Example 2

\begin{tabular}{|c|c|c|c|c|c|c|}
\hline \multirow{2}{*}{ Item } & \multicolumn{2}{|c|}{$\begin{array}{c}\text { Design } \\
\text { parameters }\end{array}$} & \multirow{2}{*}{$\begin{array}{l}\text { Cost, } \\
C\left(\mathrm{~m}^{3}\right)\end{array}$} & \multirow{2}{*}{$\begin{array}{c}\text { Sensitivity } \\
\text { index, } \\
\text { SI }\end{array}$} & \multicolumn{2}{|c|}{$\begin{array}{c}\text { Normalized } \\
\text { objectives }\end{array}$} \\
\hline & $B(\mathrm{~m})$ & $D(\mathrm{~m})$ & & & $C^{\mathrm{n}}$ & $\mathrm{SI}^{\mathrm{n}}$ \\
\hline Least cost design $^{(1)}$ & 0.30 & 20.30 & 1.43 & 0.465 & 0.0 & 1.0 \\
\hline Most robust design $^{(2)}$ & 0.60 & 25.00 & 7.07 & 0.352 & 1.0 & 0.0 \\
\hline Most preferred design ${ }^{(3)}$ & 0.37 & 25.00 & 2.66 & 0.383 & 0.217 & 0.273 \\
\hline
\end{tabular}

Note:

(1) The least cost design is identified by minimizing the cost $(C)$.

(2) The most robust design is identified by minimizing the sensitivity index (SI).

(3) The most preferred design is identified by minimizing the distance measured from the utopia design to each of the candidate designs. 
Table 5. Comparison of the computational efficiency between the simplified procedure and the existing multi-objective optimization procedure

\begin{tabular}{|c|c|c|}
\hline Adopted approach & Analysis steps & $\begin{array}{c}\text { Computation time } \\
\text { (second) }\end{array}$ \\
\hline \multirow{3}{*}{ Proposed procedure } & 1) Locate the least cost design ${ }^{(1)}$ & 0.0200 \\
\hline & 2) Locate the most robust design ${ }^{(1)}$ & 0.0080 \\
\hline & 3) Locate the most preferred design ${ }^{(1)}$ & 0.0140 \\
\hline \multirow[b]{2}{*}{ Existing procedure } & 1) Locate the Pareto front with NSGA-II ${ }^{(2)}$ & 68.1057 \\
\hline & $\begin{array}{l}\text { 2) Locate the knee point using marginal } \\
\text { utility function approach }\end{array}$ & 7.9290 \\
\hline
\end{tabular}

Note:

(1) The optimization setting is set at: options=optimset('Algorithm','active-set','LargeScale', 'on','Display','off', 'TolFun',1e-3,'MaxIter',50,'MaxFunEvals',1e7,'TolX',1e-3,'TolCon',1e-3).

(2) The population size and generation number in NSGA_II are set at 100 and 20,000, respectively; and, four parallel computations are utilized within Matlab.

(3) 10,000 MCS samples are employed for evaluating the marginal utility function. 


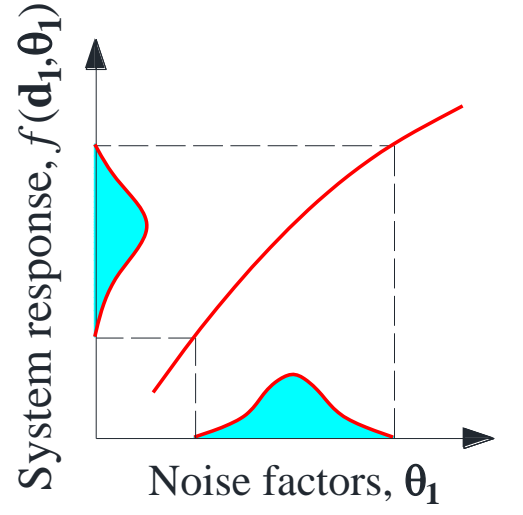

(a)

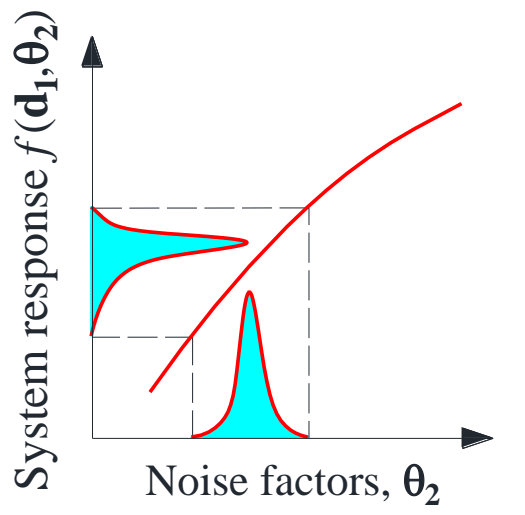

(b)

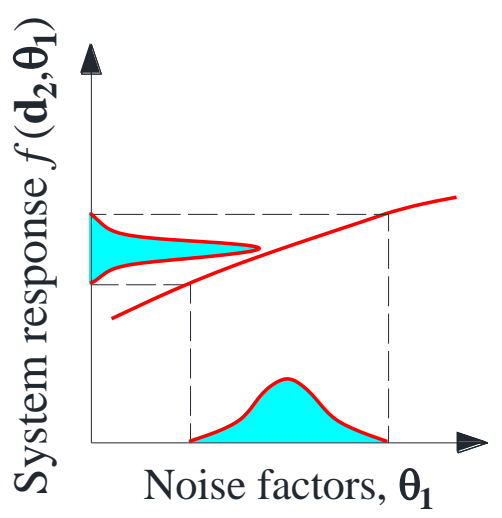

(c)

Figure 1. Variation in the system response: (a) System response of a non-robust design $\mathbf{d}_{1}$ with noise factors as its input; (b) Reducing the variation in the system response by reducing the variation in noise factors; (c) Reducing the variation in the system response by adopting a robust design $\mathbf{d}_{\mathbf{2}}$ without reducing the variation in noise factors. 


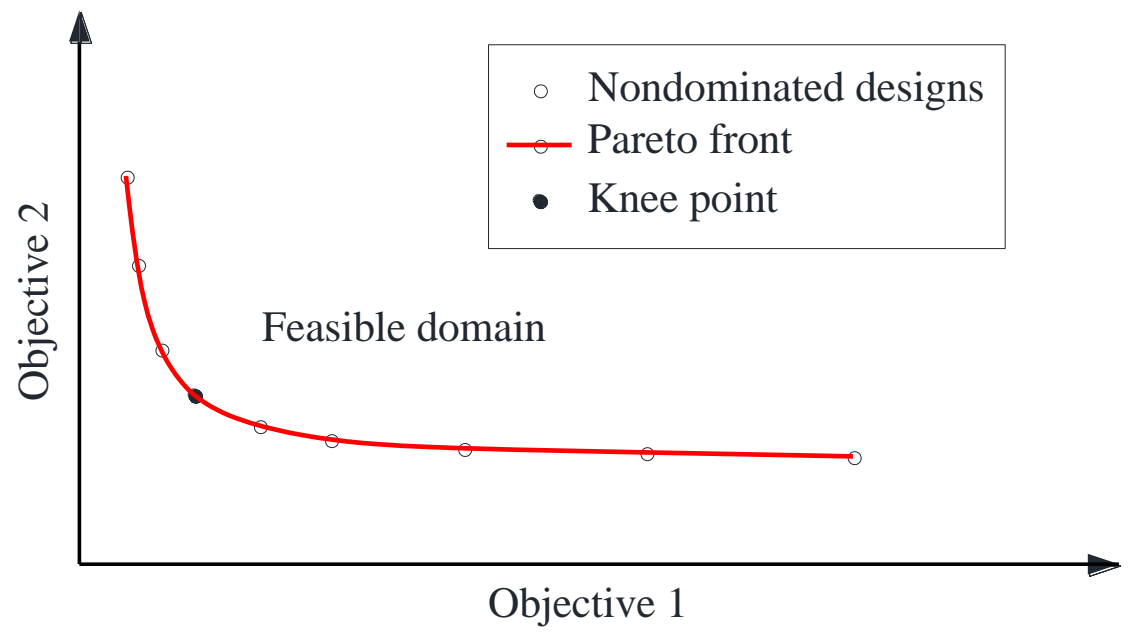

(a) Pareto front and knee point

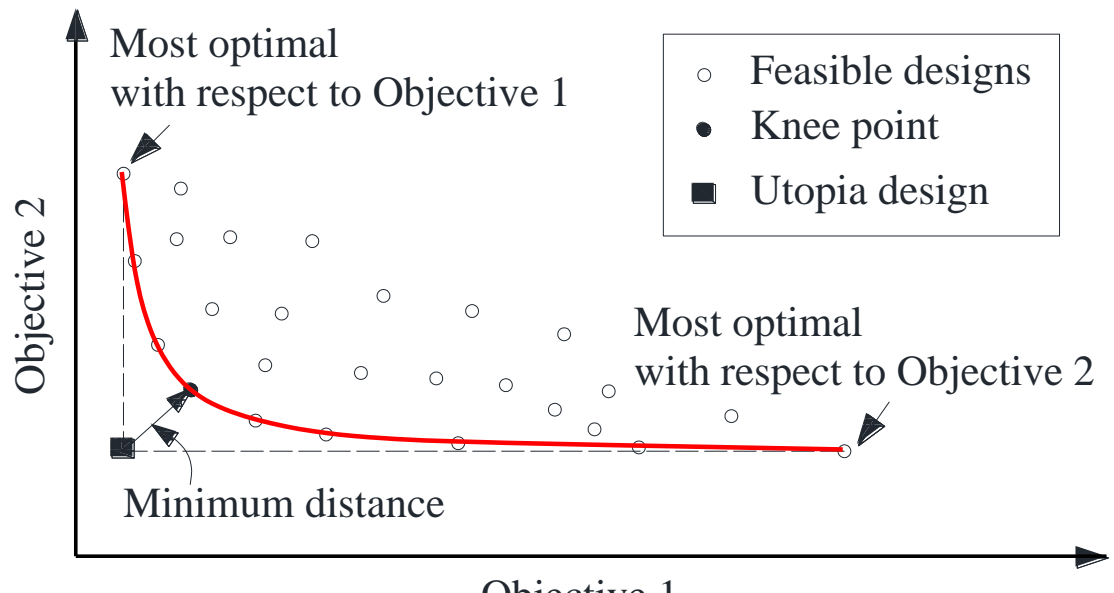

(b) Simplified procedure to identify the most preferred design in the design space

Figure 2. Conceptual illustration of a bi-objective optimization problem 


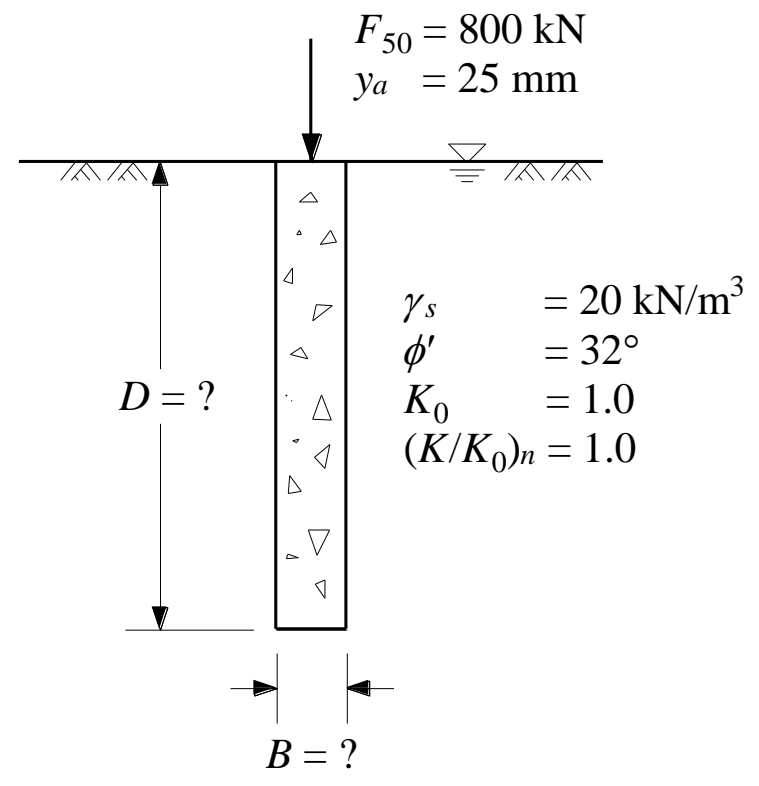

Figure 3. Schematic diagram of the design of a drilled shaft in sand (Example 1) 


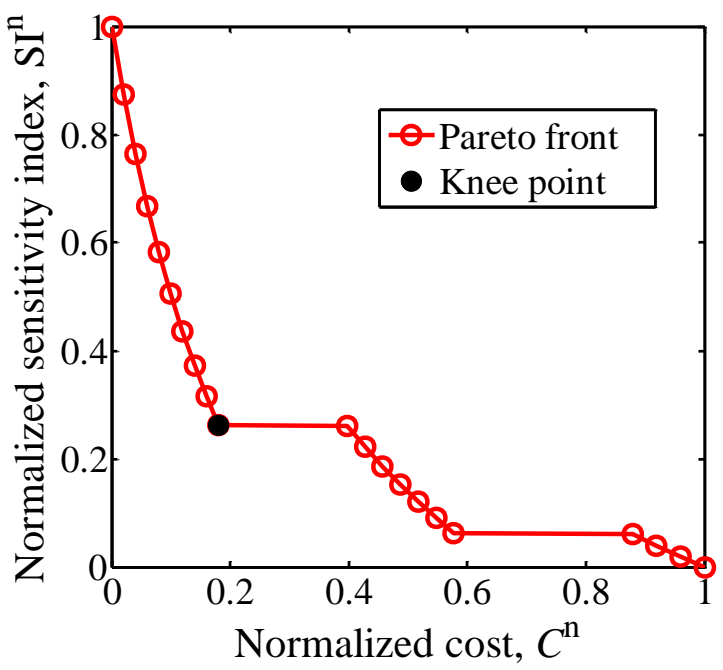

(a) Normalized design objectives

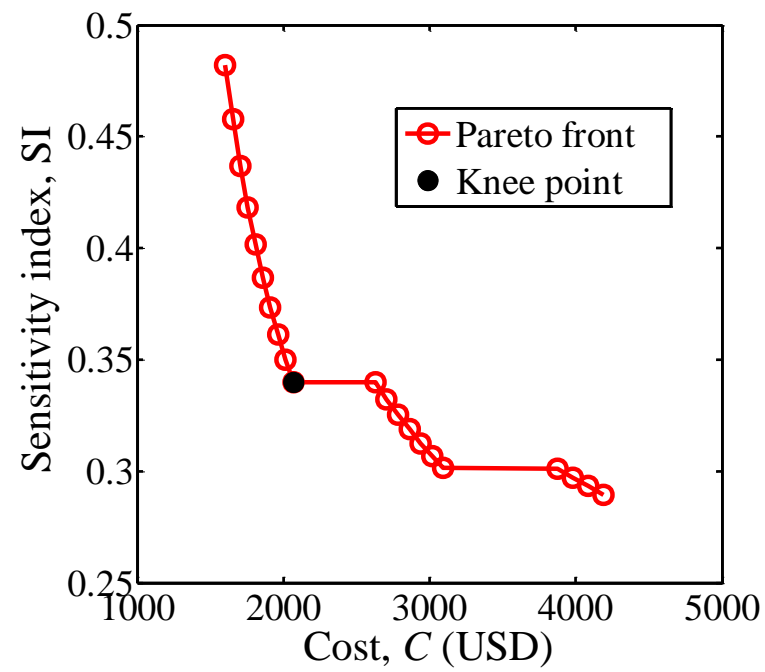

(b) Unnormalized design objectives

Figure 4. The Pareto front and knee point obtained for the drilled shaft in sand (Example 1) 


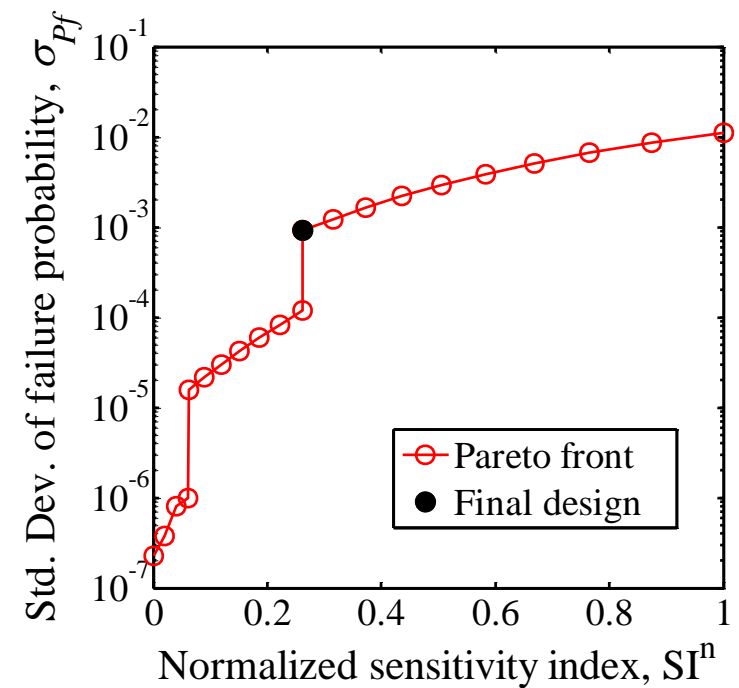

(a) $\sigma_{P_{f}}$ versus $\mathrm{SI}^{\mathrm{n}}$

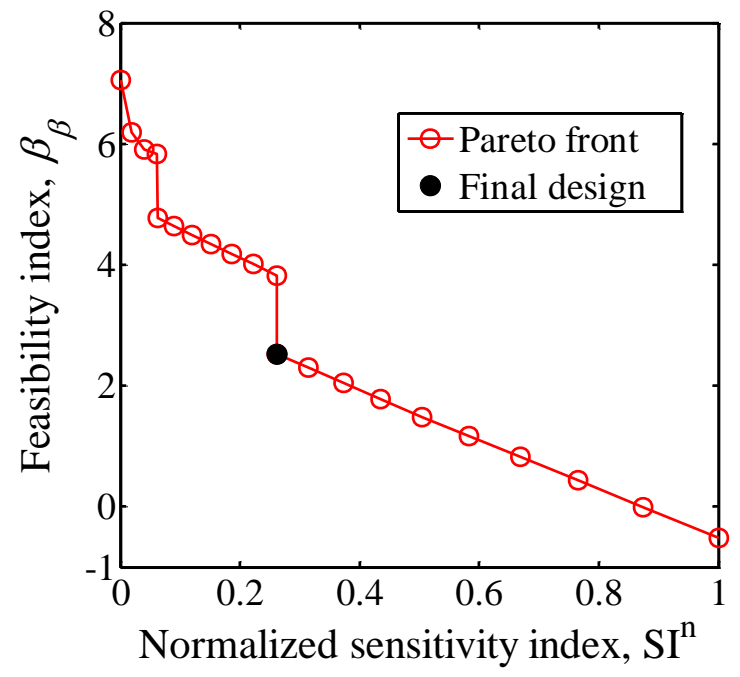

(b) $\beta_{\beta}$ versus $\mathrm{SI}^{\mathrm{n}}$

Figure 5. Effectiveness of the sensitivity index as a robustness measure (Example 1) 


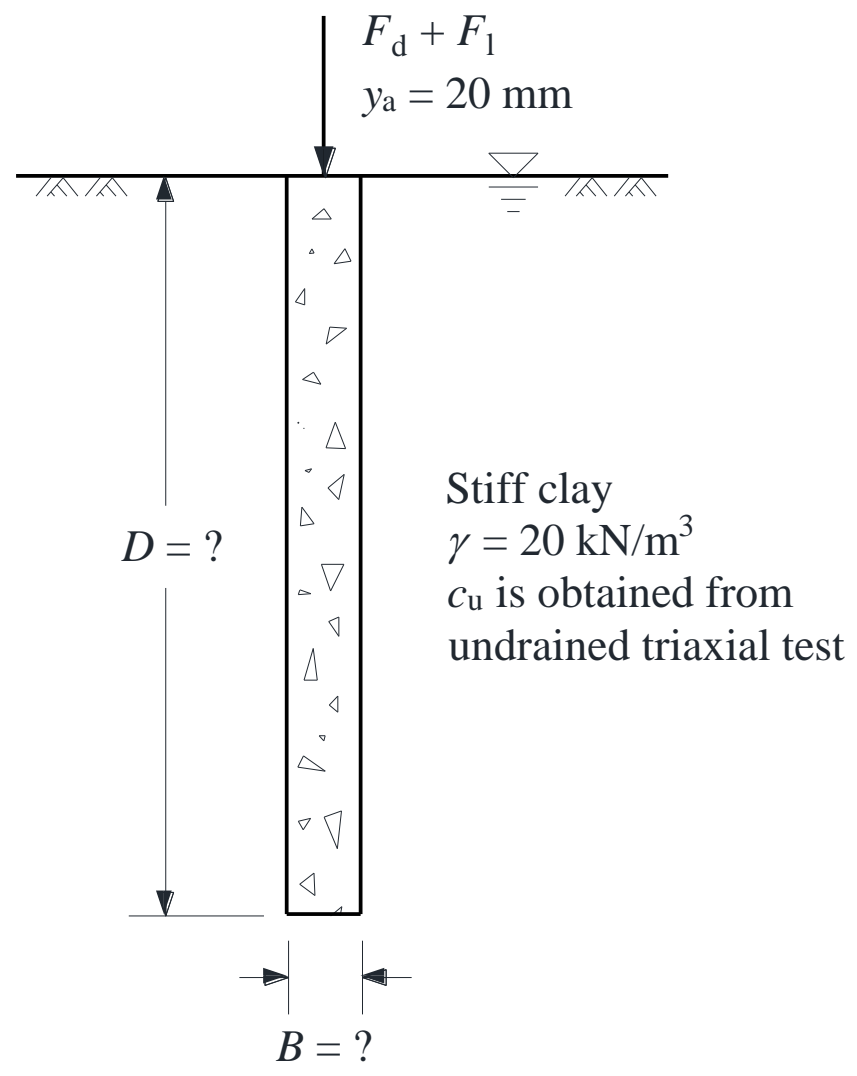

Figure 6. Schematic diagram of the design of a drilled shaft in clay (Example 2) 


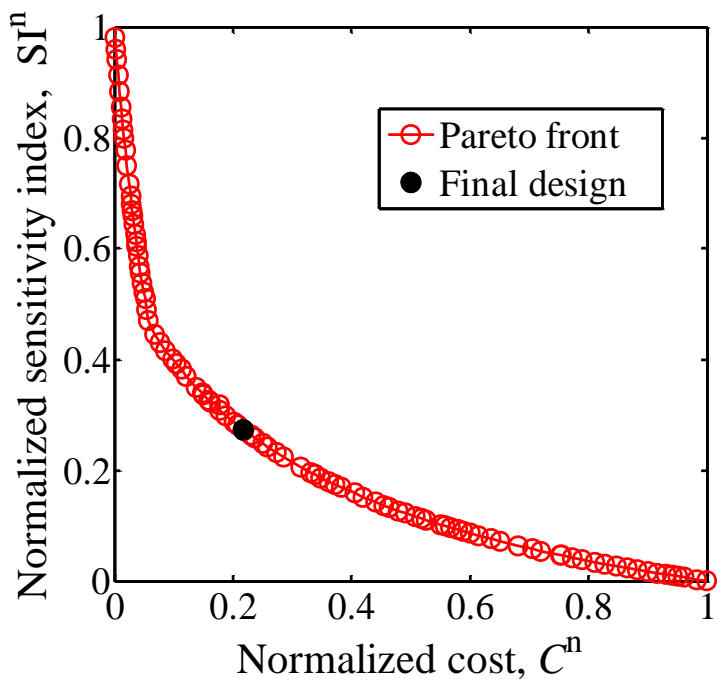

(a) Normalized design objectives

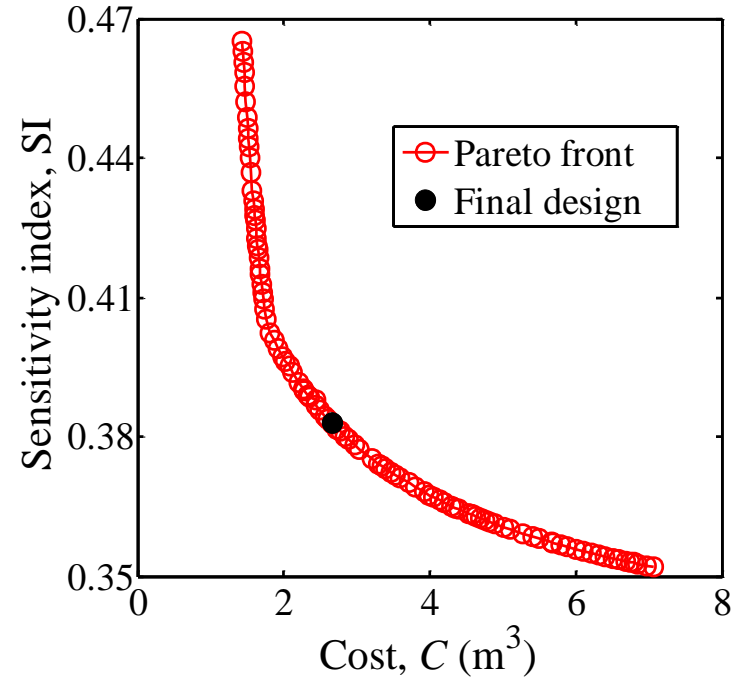

(b) Unnormalized design objectives

Figure 7. The Pareto front and knee point obtained for the drilled shaft in clay (Example 2) 


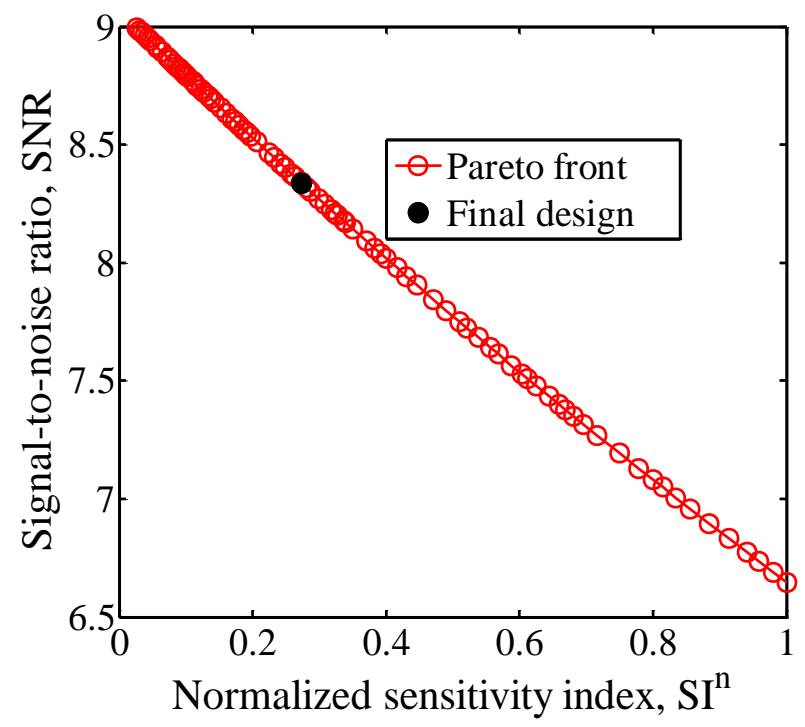

Figure 8. Effectiveness of the sensitivity index as a robustness measure (Example 2) 


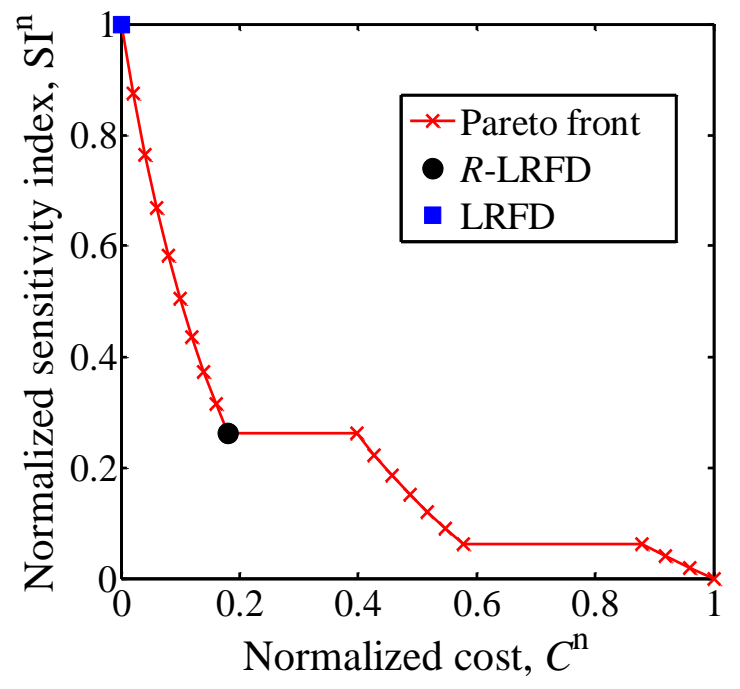

(a) Example 1

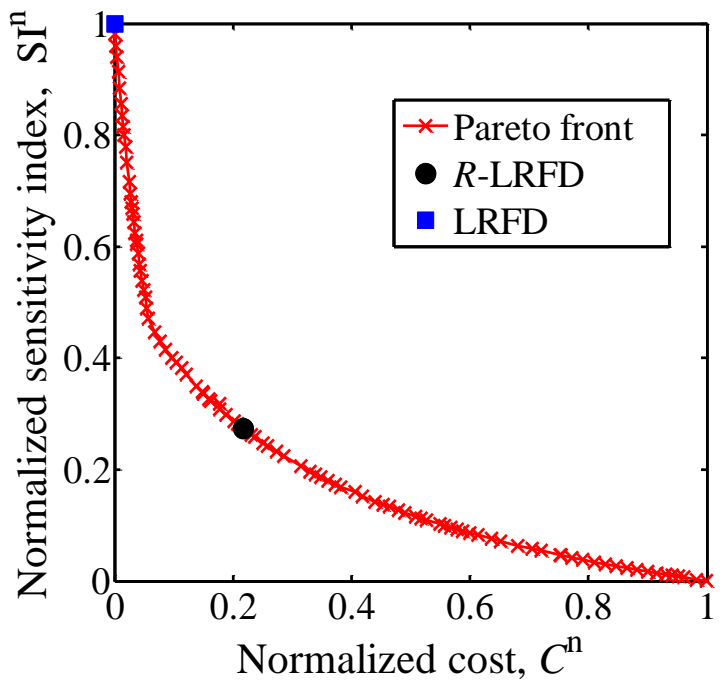

(b) Example 2

Figure 9. Comparison of designs obtained with LRFD and $R$-LRFD 